\title{
The impact of MISR-derived injection height initialization on wildfire and volcanic plume dispersion in the HYSPLIT model
}

\section{Charles J. Vernon et al.}

Correspondence to: Charles J. Vernon (c.vernon1414@gmail.com) 


\section{Supplemental Material}

2

3

4
Figure S1

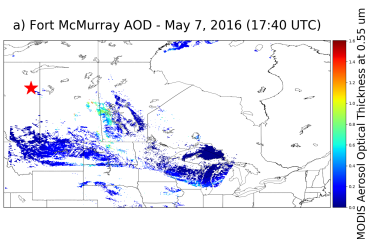

d) Eyjafjallajokull AOD - May 9, 2010 (12:25 UTC)

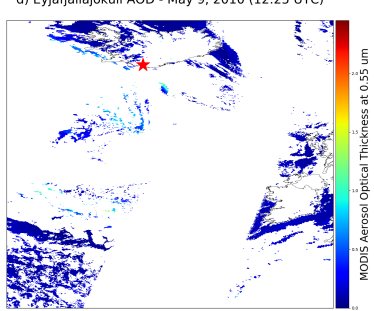

MODIS Aerosol Optical Depth (AOD)
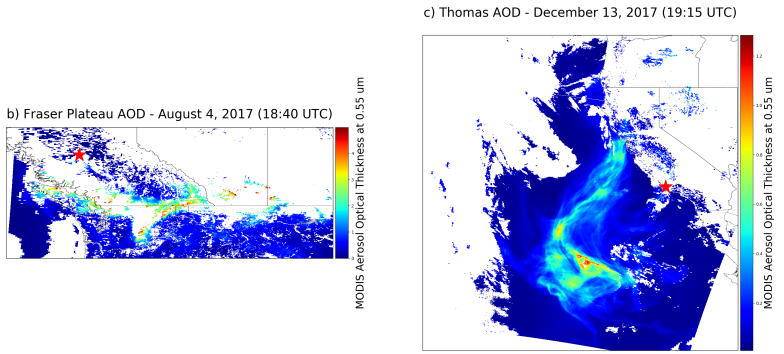

f) Chikurachki AOD - April 25, 2003 (01:15 UTC)
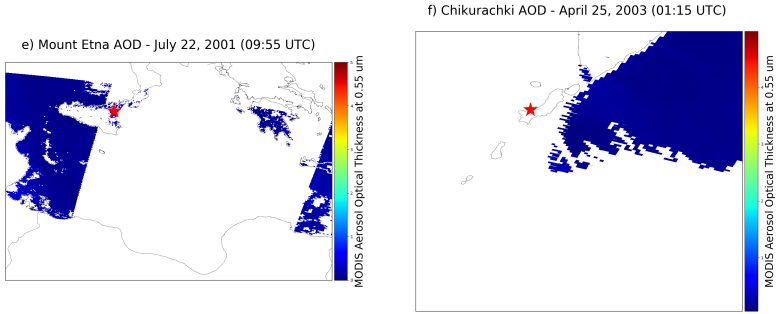

\section{Yosemite Rim Fire Plume, August 2013}

The Yosemite Rim Fire was also one of the simulations performed for this study but is temporarily being left in supplemental material. It is possible that the method used to convert BlueSky data into a HYSPLIT compatible format or the BlueSky data itself could be inaccurate since there was an older version of BlueSky in 2013. We are currently waiting on confirmation from the National Center for Environmental Prediction about whether we can call this simulation nominal. The analysis of the simulations that were performed can be found below.

August $23^{\text {rd }}, 2013$, Day 1 of our Yosemite Rim Fire simulations, shows noticeable differences between the nominal and MISR-initialized plumes. Figure $2 \mathrm{~b}$ shows the sounding at KRNO (August $24^{\text {th }}, 201300$ UCT) just north of Yosemite, which indicates that the PBL height is approximately 4.5 kilometers. The GDAS meteorological fields The MINX injection height was 6.2 kilometers and the HYSPLIT calculation placed the injection height at 3 kilometers. This case again indicates the importance of accurately initializing the smoke plume height, especially when it is above the top of the boundary layer. The wind shifts just above the PBL changing from 10 knots out of the west to 15 20 knots out of the south. As the nominal HYSPLIT simulation injects the smoke below the height where this occurs, Figure 4 shows that the plume does not extend nearly as far downwind as the MISR-initialized plume. In this case, the MISR-initialized plume reaches from the United States north beyond the border with Canada. Both the optical depth map and the visible imagery show a well defined, optically thick smoke plume 
1 extending from Yosemite past the border and then looping back to the southeast. The 2 nominal model simulation has visible smoke extending only to the southern border of 3 Idaho, whereas the MISR-initialized run shows visible smoke reaching the border, in 4 much better agreement with observations.

5

6

7

8

9

10

11

12

13
4) Yosemite Rim Fire Simulation - August 23, 2013 (18:55 UTC)
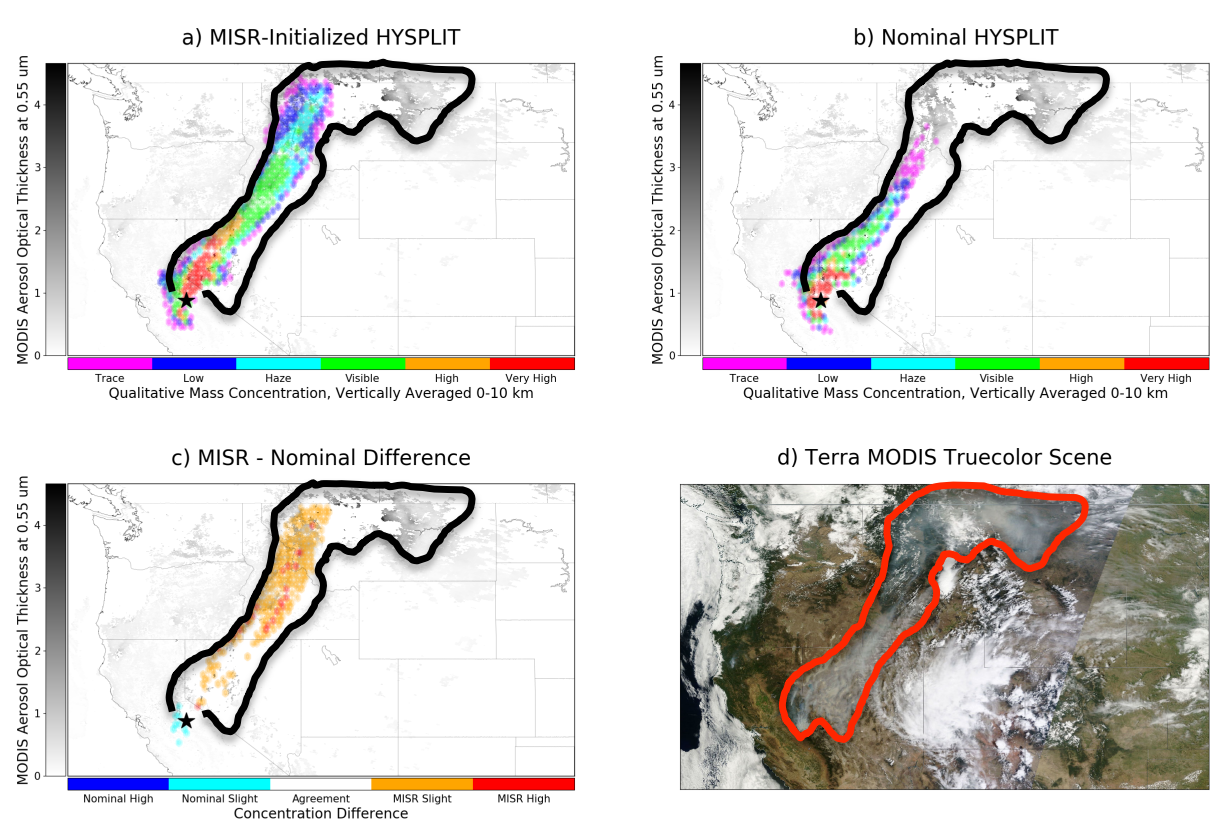

\section{Figure S2}

$14 \quad$ Figure S3

\section{Additional Figures}

3 Yosemite 
Yosemite Rim Fire Simulation - August 24, 2013 (21:15 UTC)
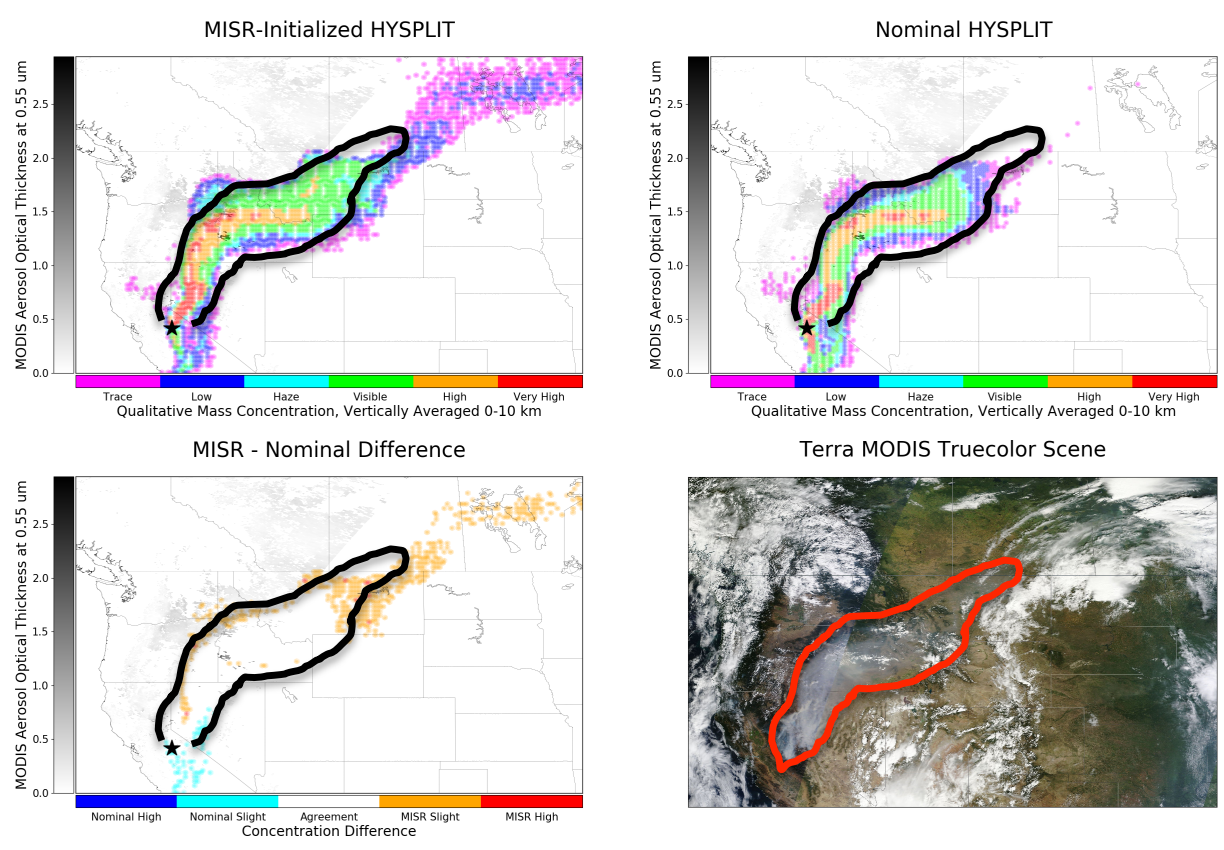

Yosemite Rim Fire Simulation - August 25, 2013 (18:45 UTC)
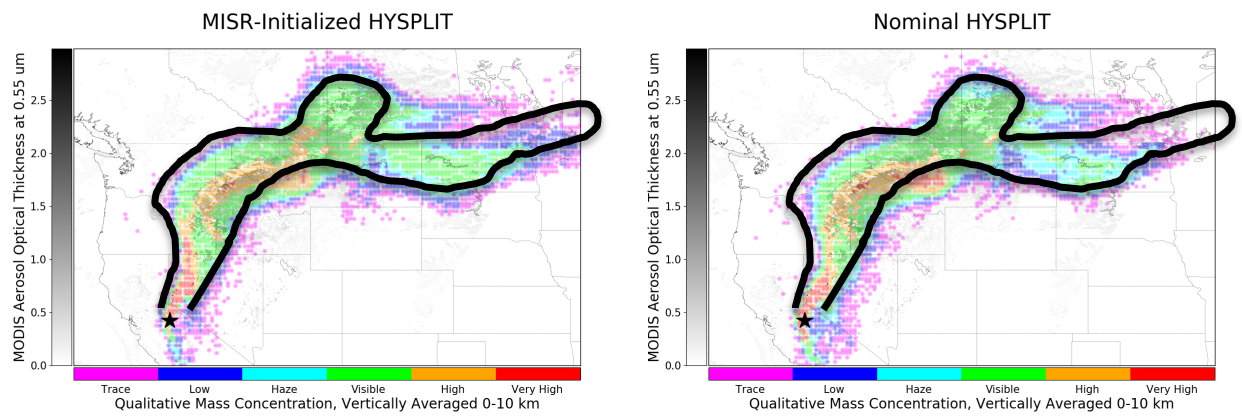

MISR - Nominal Difference

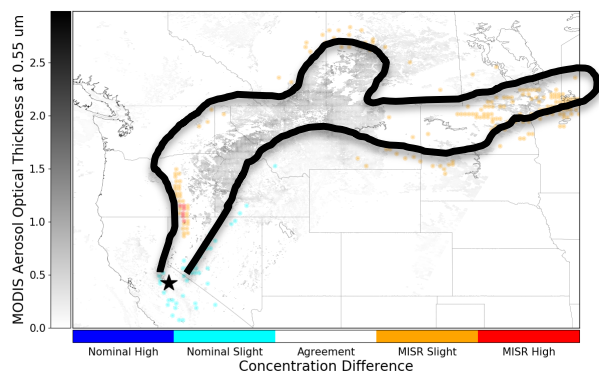

Terra MODIS Truecolor Scene

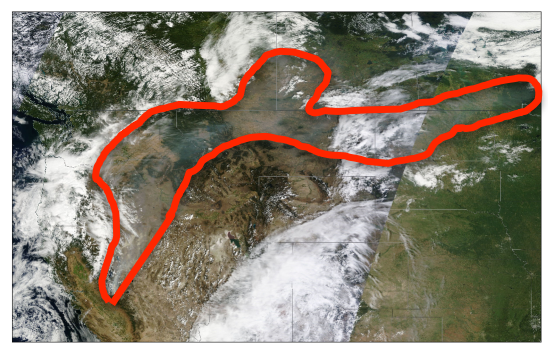


Yosemite Rim Fire Simulation - August 26, 2013 (19:25 UTC)
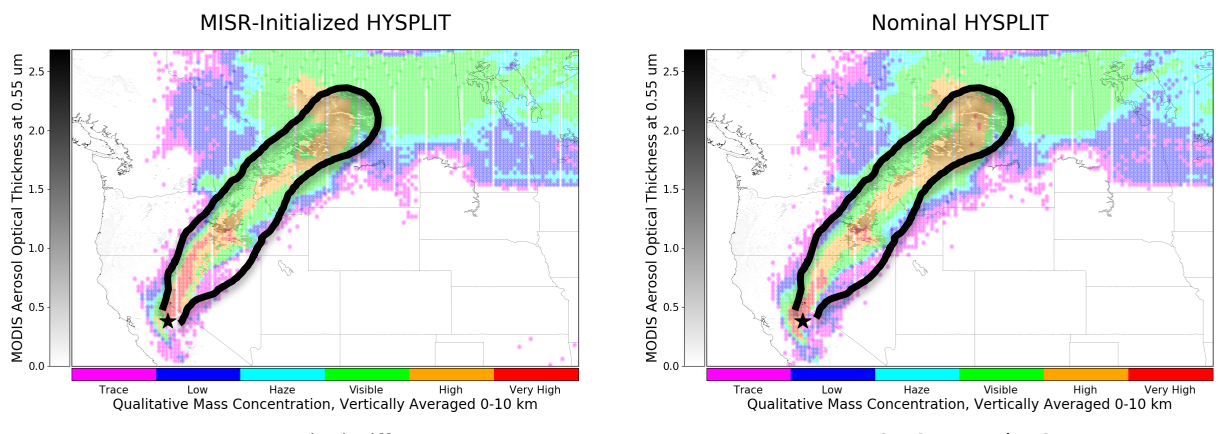

MISR - Nominal Difference

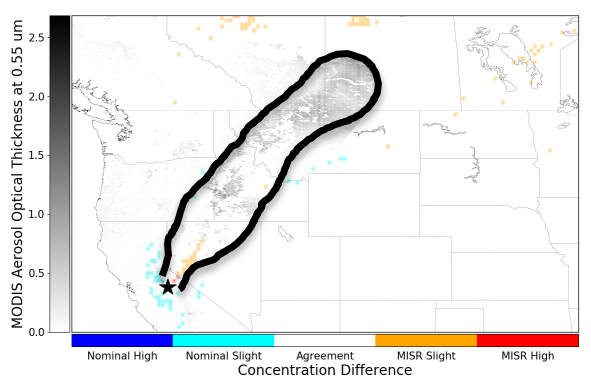

Terra MODIS Truecolor Scene

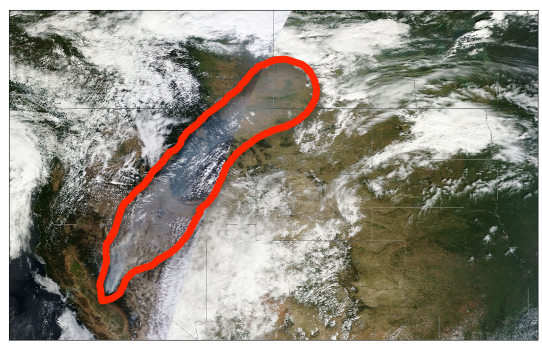

1

3 Fort McMurray

$4 \quad$ Figure S6

Fort McMurray Wildfire Simulation - May 6, 2016 (18:35 UTC)
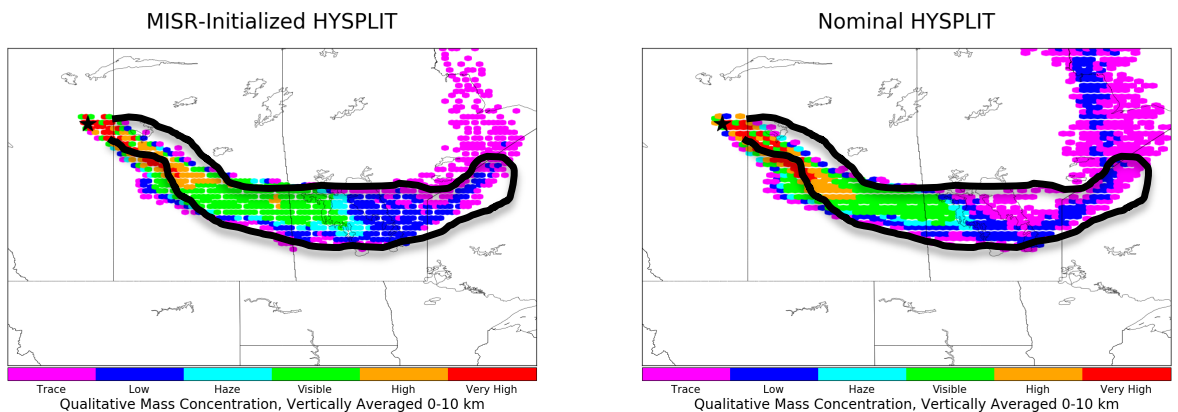

MISR - Nominal Difference

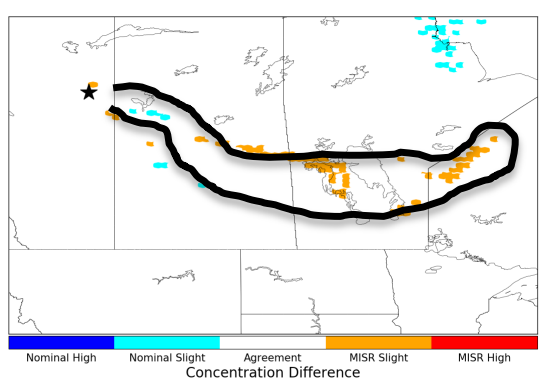

Terra MODIS Truecolor Scene

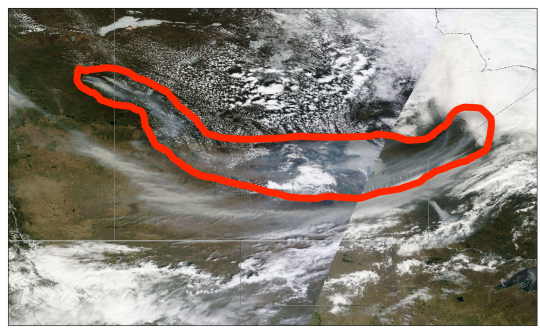


Fort McMurray Wildfire Simulation - May 8, 2016 (18:20 UTC)
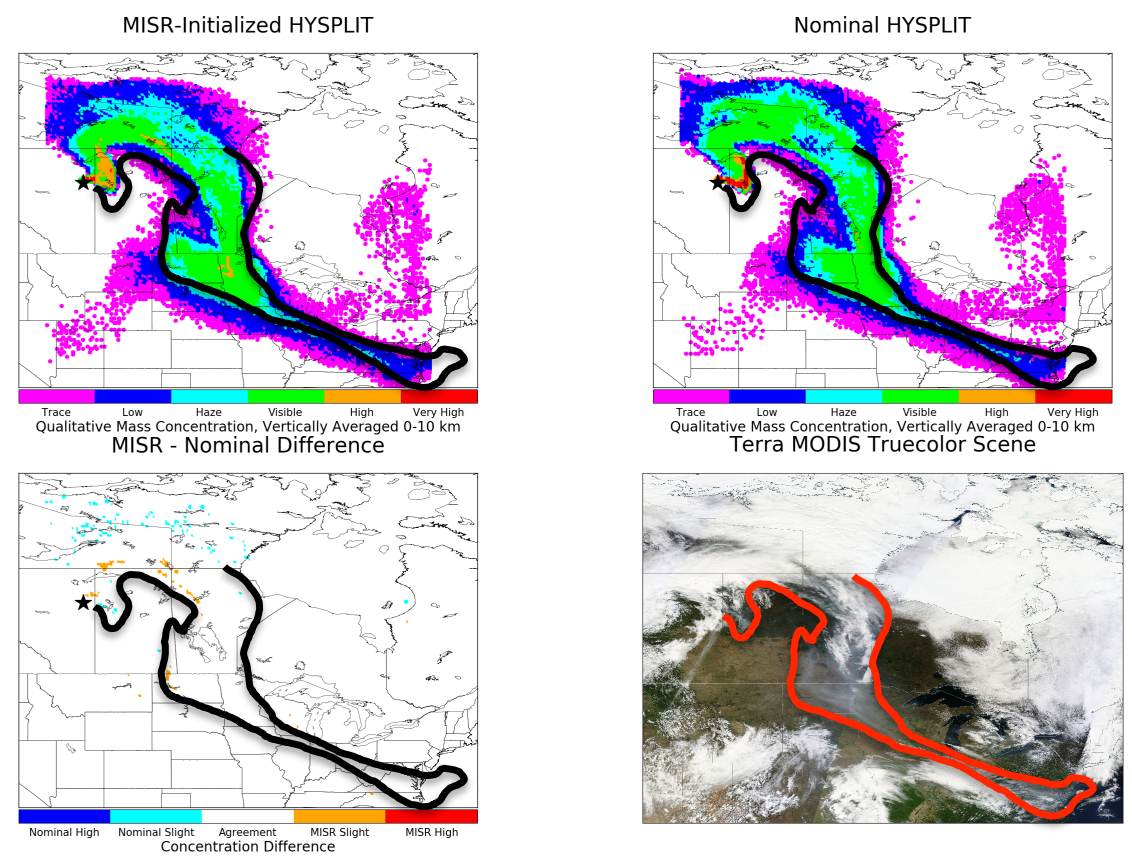

Figure S8

Fort McMurray Wildfire Simulation - May 9, 2016 (19:10 UTC)
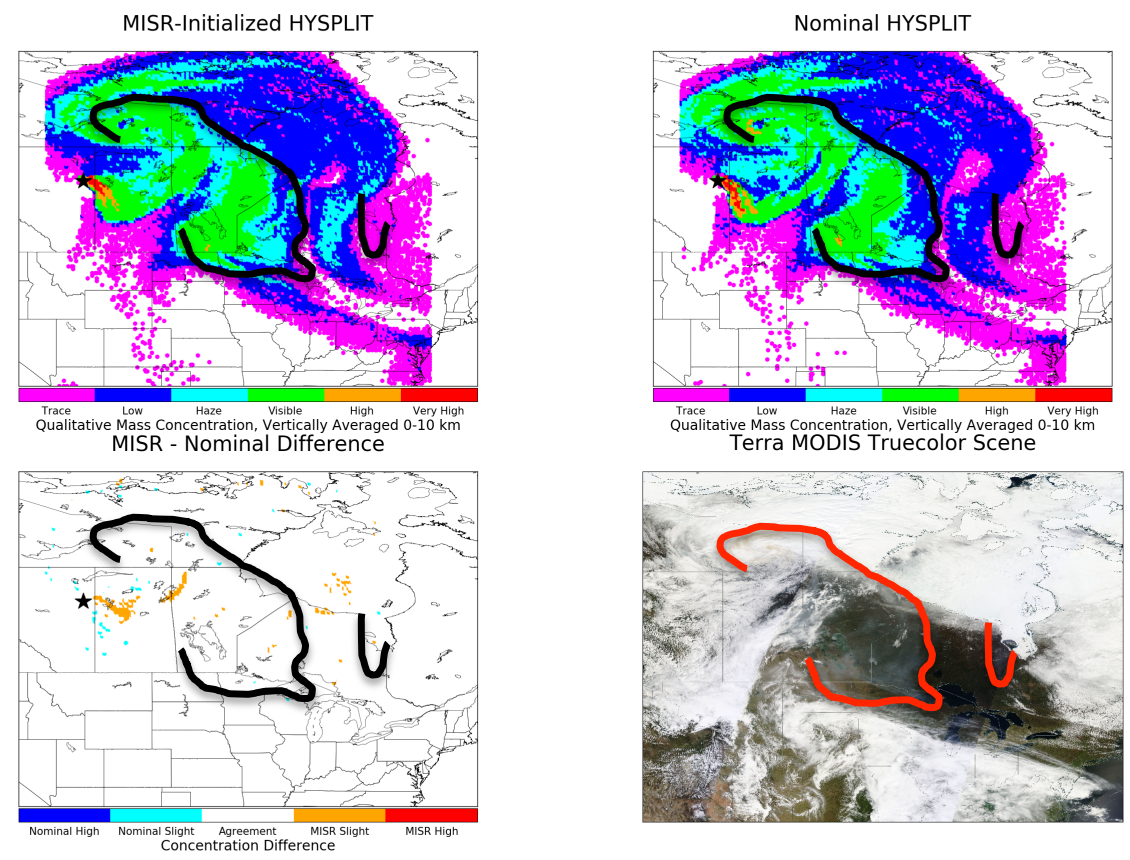
Fraser Plateau Wildfire Simulation - August 3, 2017 (19:35 UTC)
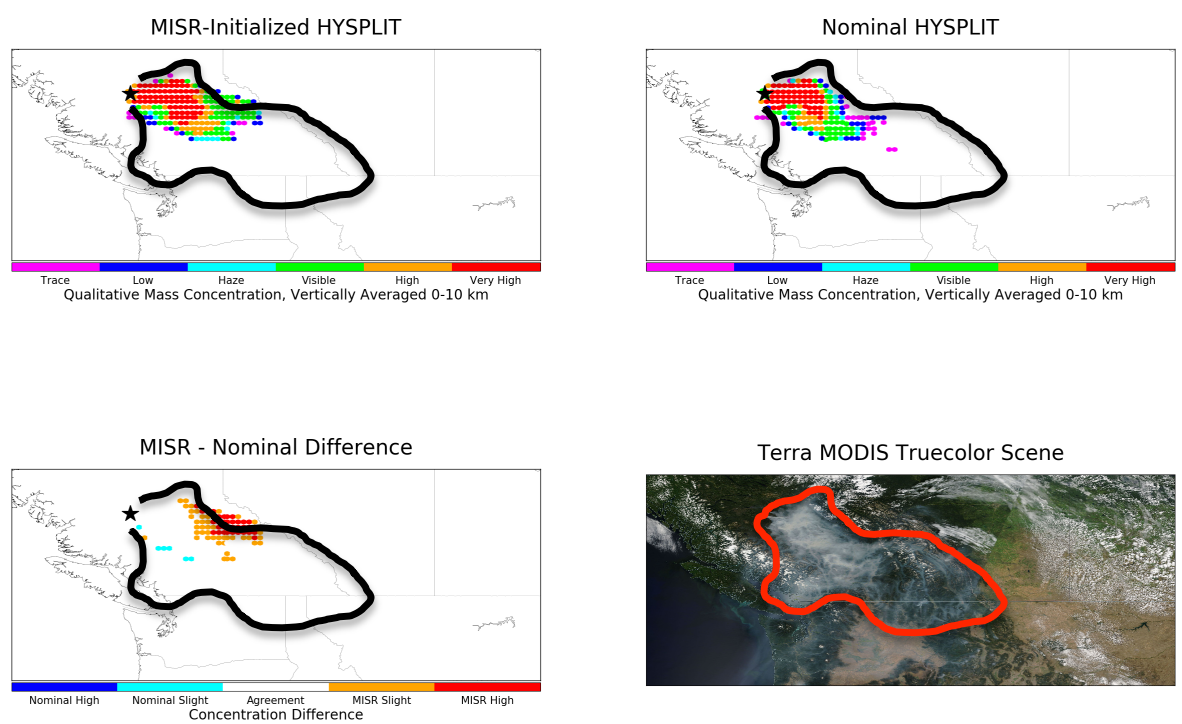

Fraser Plateau Wildfire Simulation - August 5, 2017 (21:10 UTC)
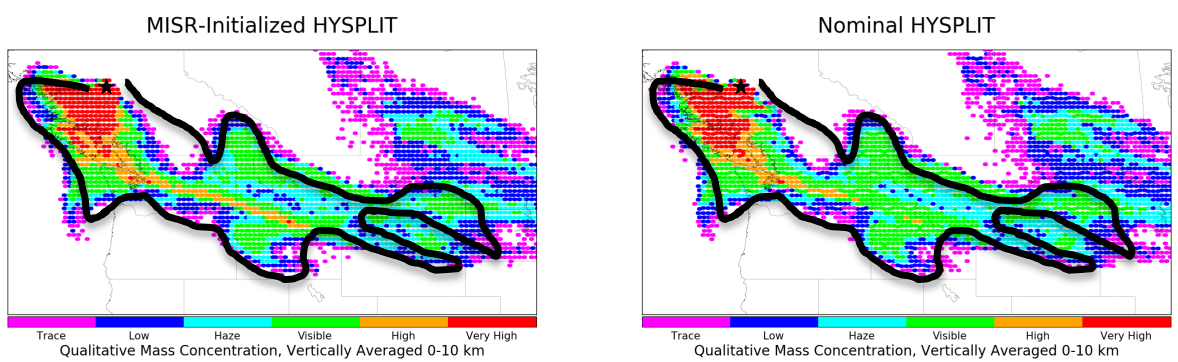

MISR - Nominal Difference

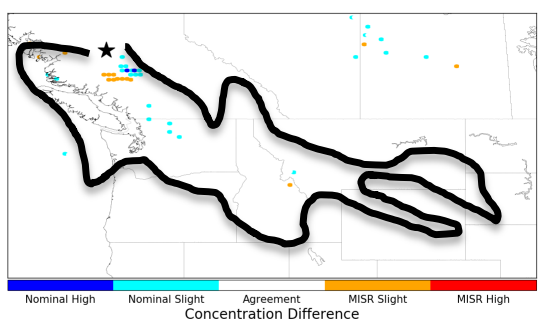

Terra MODIS Truecolor Scene

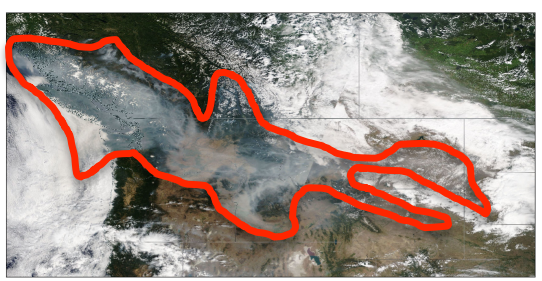

3

$4 \quad$ Figure S11 
Fraser Plateau Wildfire Simulation - August 6, 2017 (18:30 UTC)
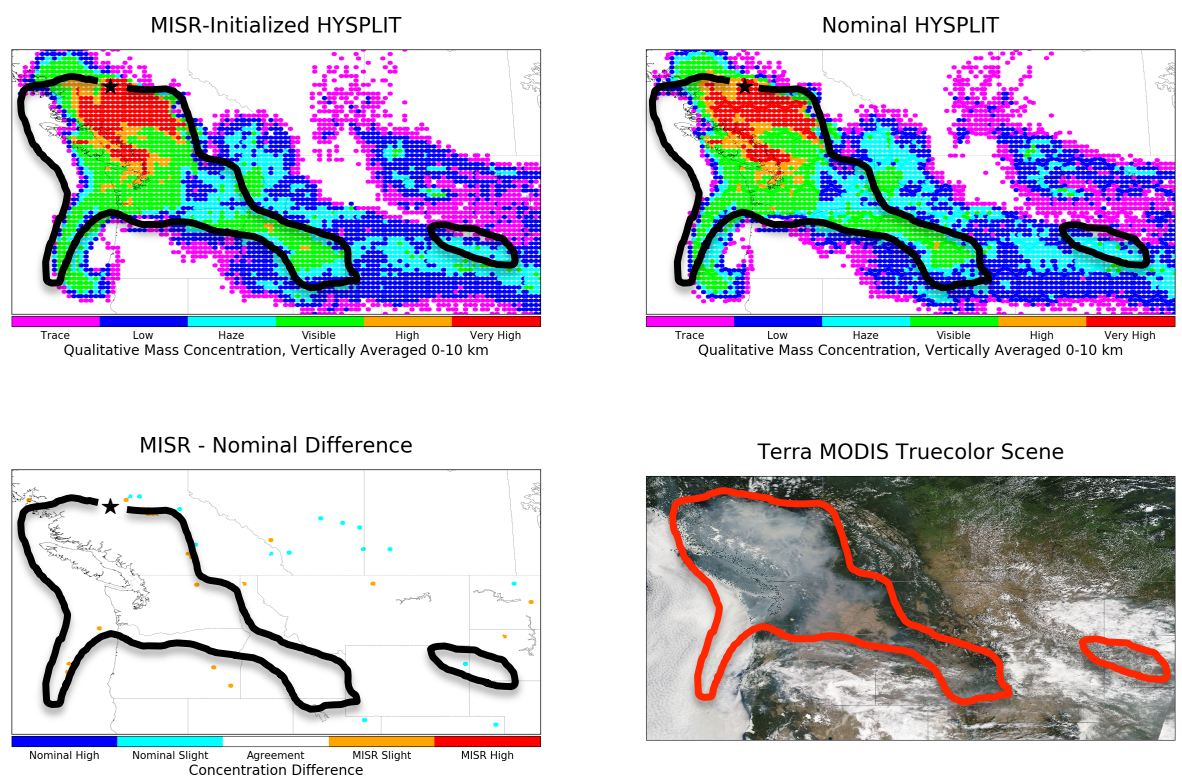

$\frac{1}{2}$

3 Thomas Fire

$4 \quad$ Figure S12 
Thomas Wildfire Simulation - December 10, 2017 (18:45 UTC)

a) MISR-Initialized HYSPLIT

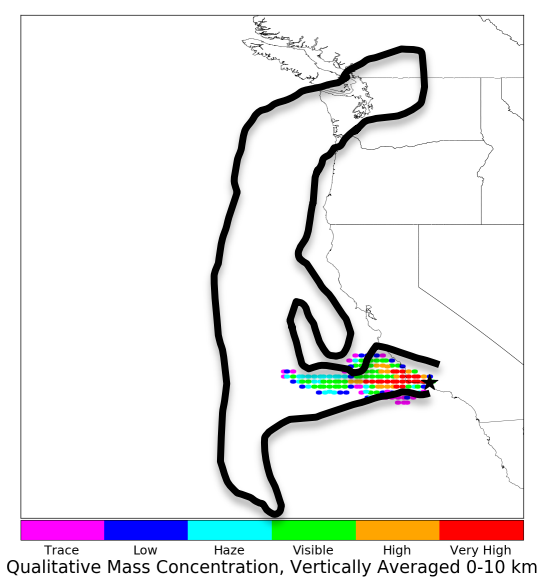

c) MISR - Nominal Difference

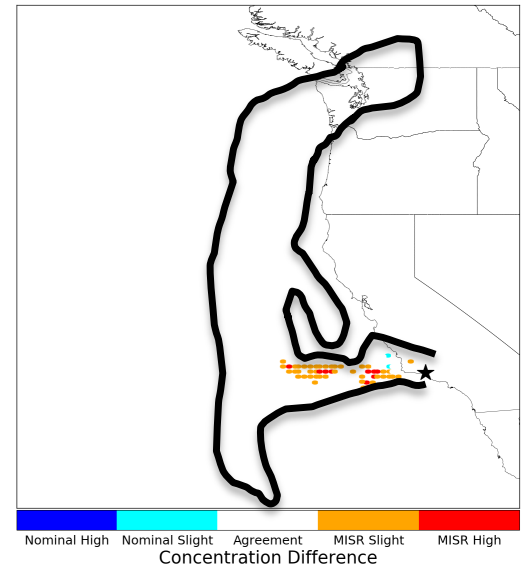

b) Nominal HYSPLIT

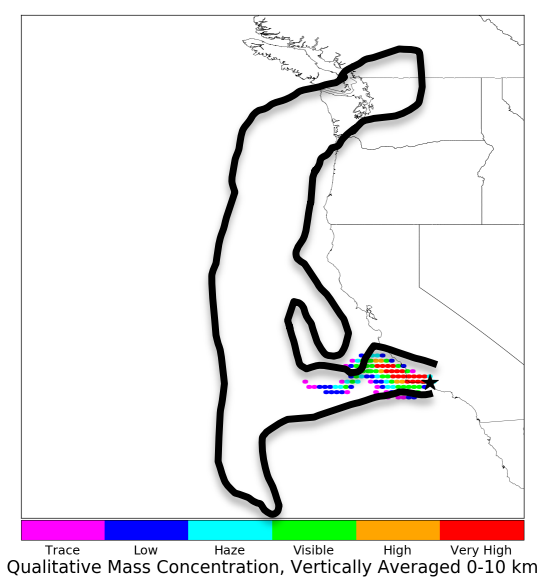

d) Terra MODIS Truecolor Scene

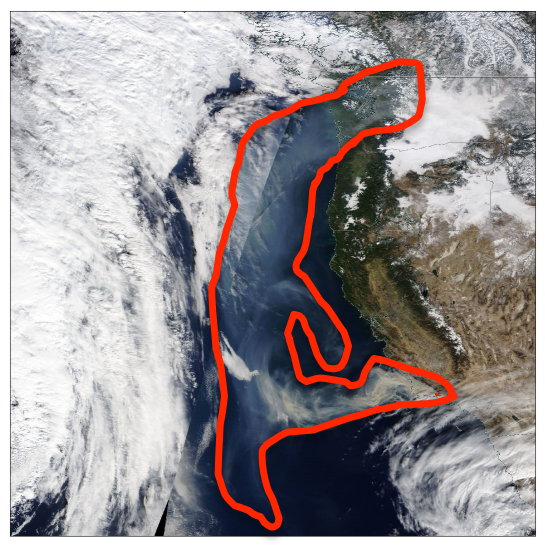


Thomas Wildfire Simulation - December 11, 2017 (19:25 UTC)
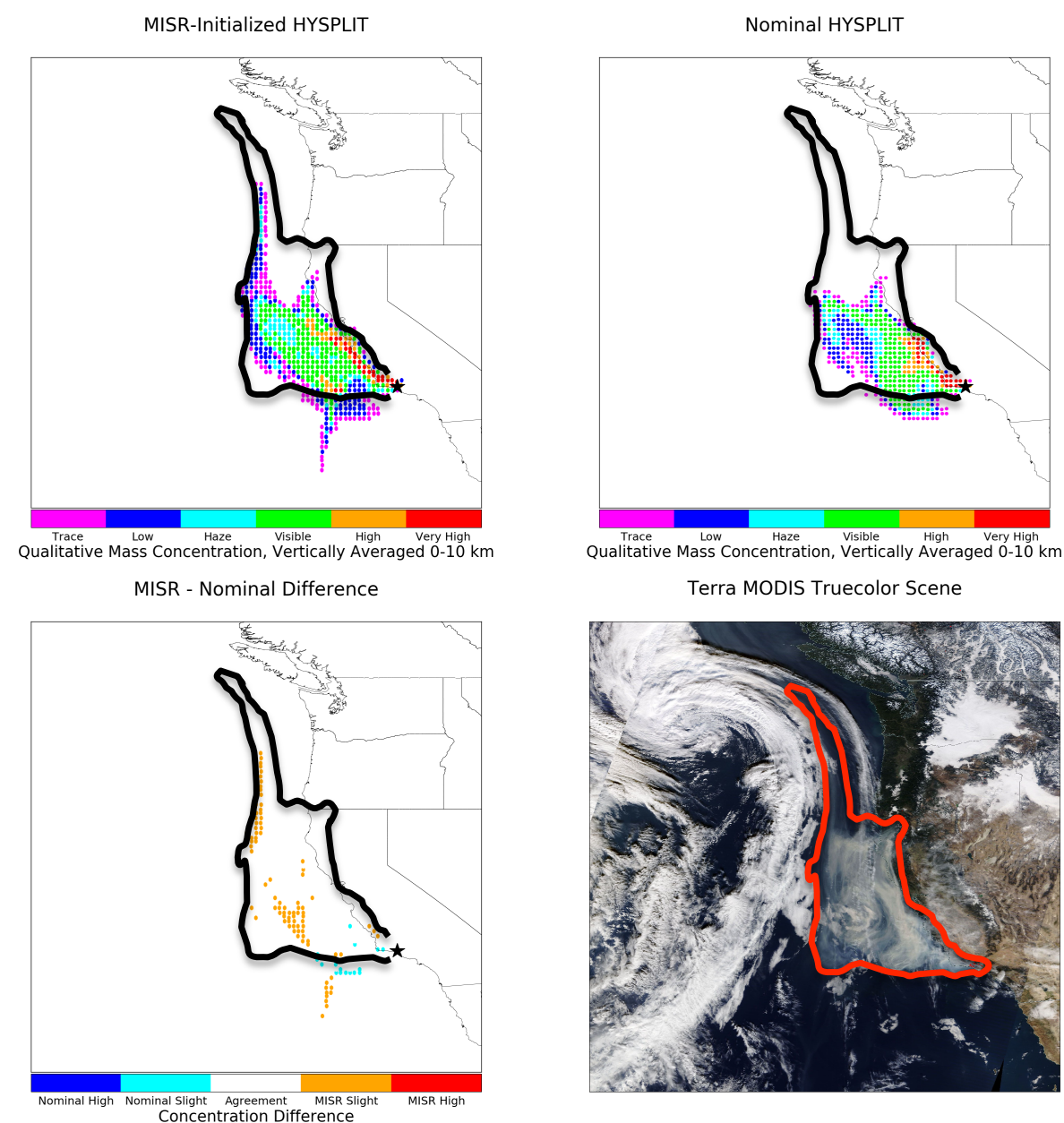
Thomas Wildfire Simulation - December 12, 2017 (18:30 UTC)
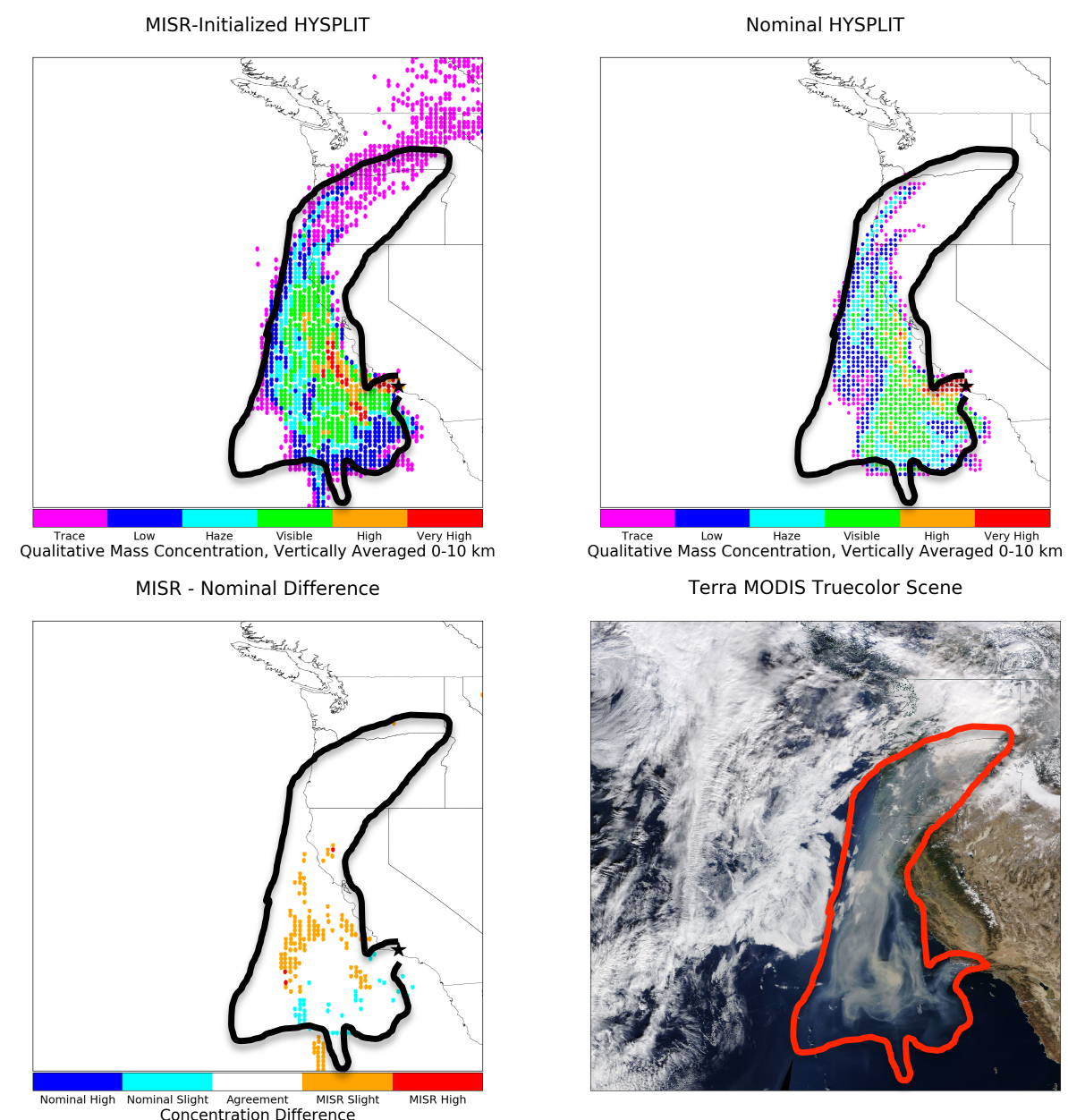

1

3 Thomas Fire with NAM12

$4 \quad$ Figure S15 
Thomas Wildfire Simulation (With NAM12) - December 10, 2017 (18:45 UTC)

a) MISR-Initialized HYSPLIT

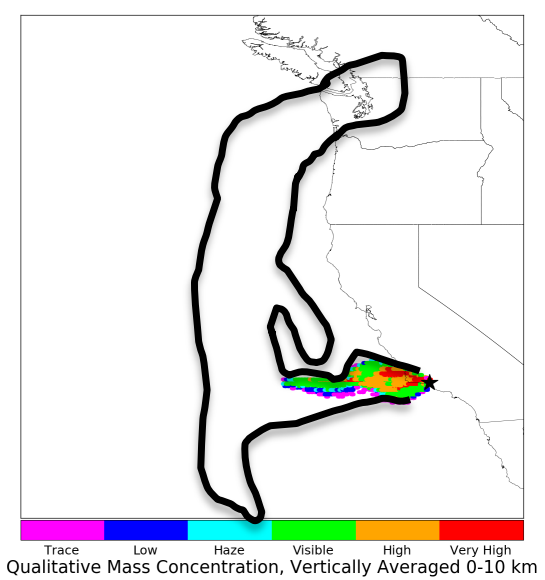

c) MISR - Nominal Difference

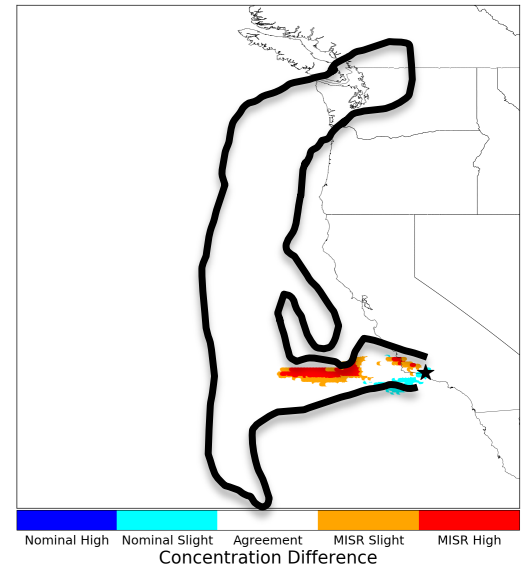

b) Nominal HYSPLIT

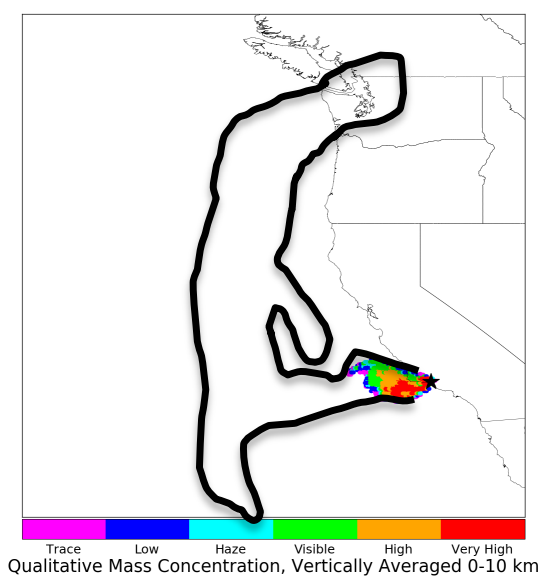

d) Terra MODIS Truecolor Scene

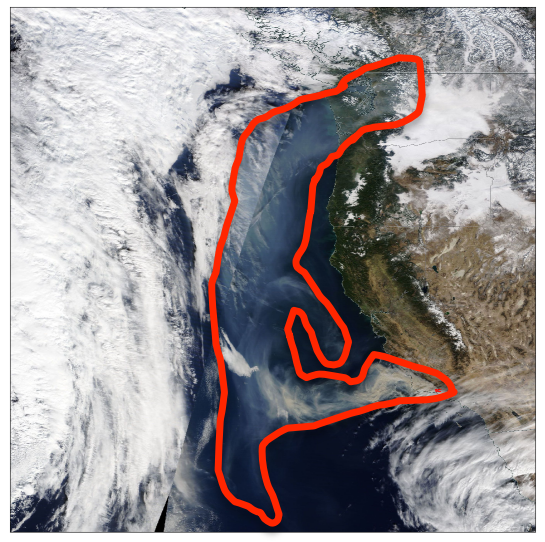


Thomas Wildfire Simulation (With NAM12) - December 11, 2017 (19:25 UTC)
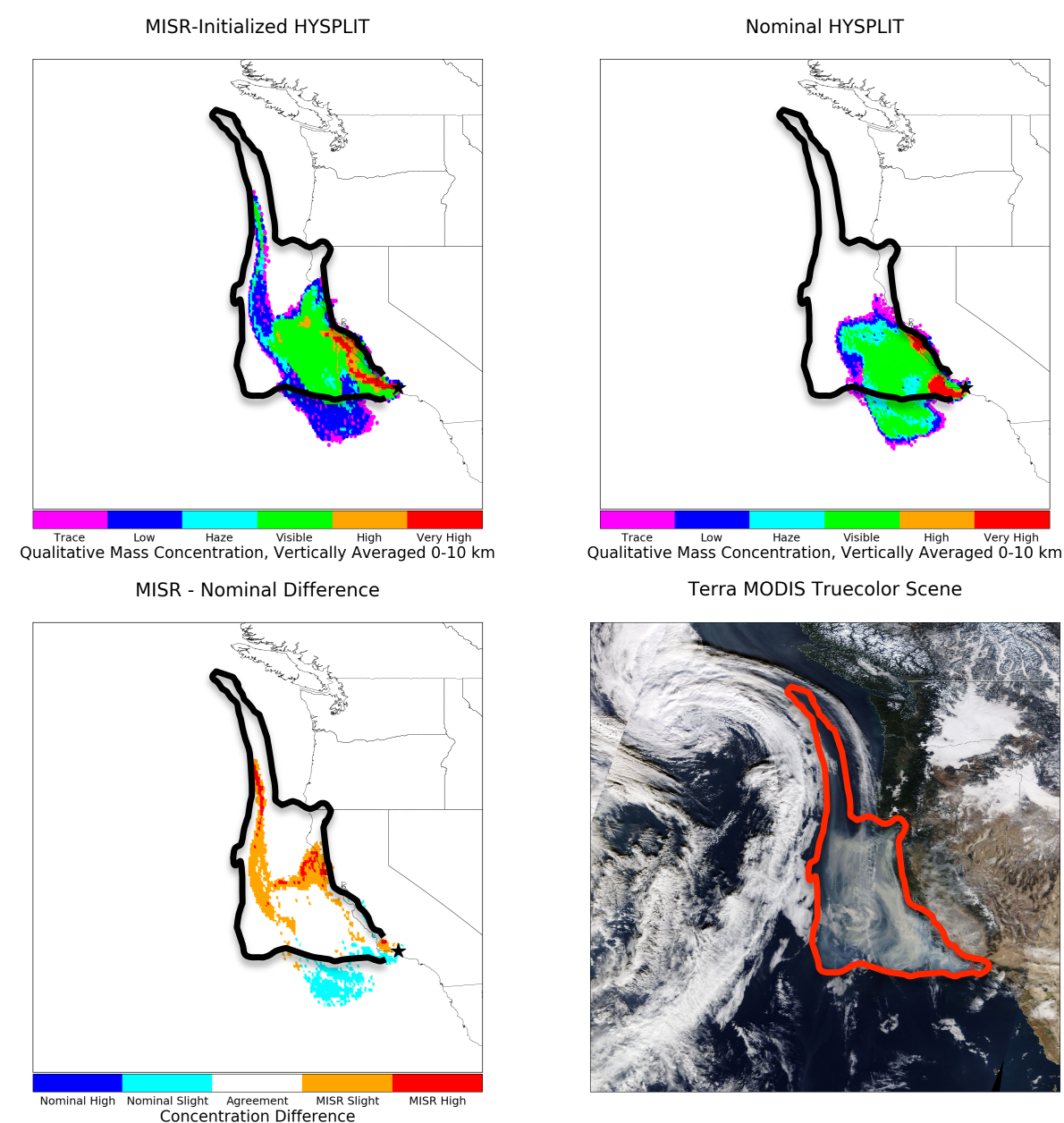
Thomas Wildfire Simulation (With NAM12) - December 12, 2017 (18:30 UTC)
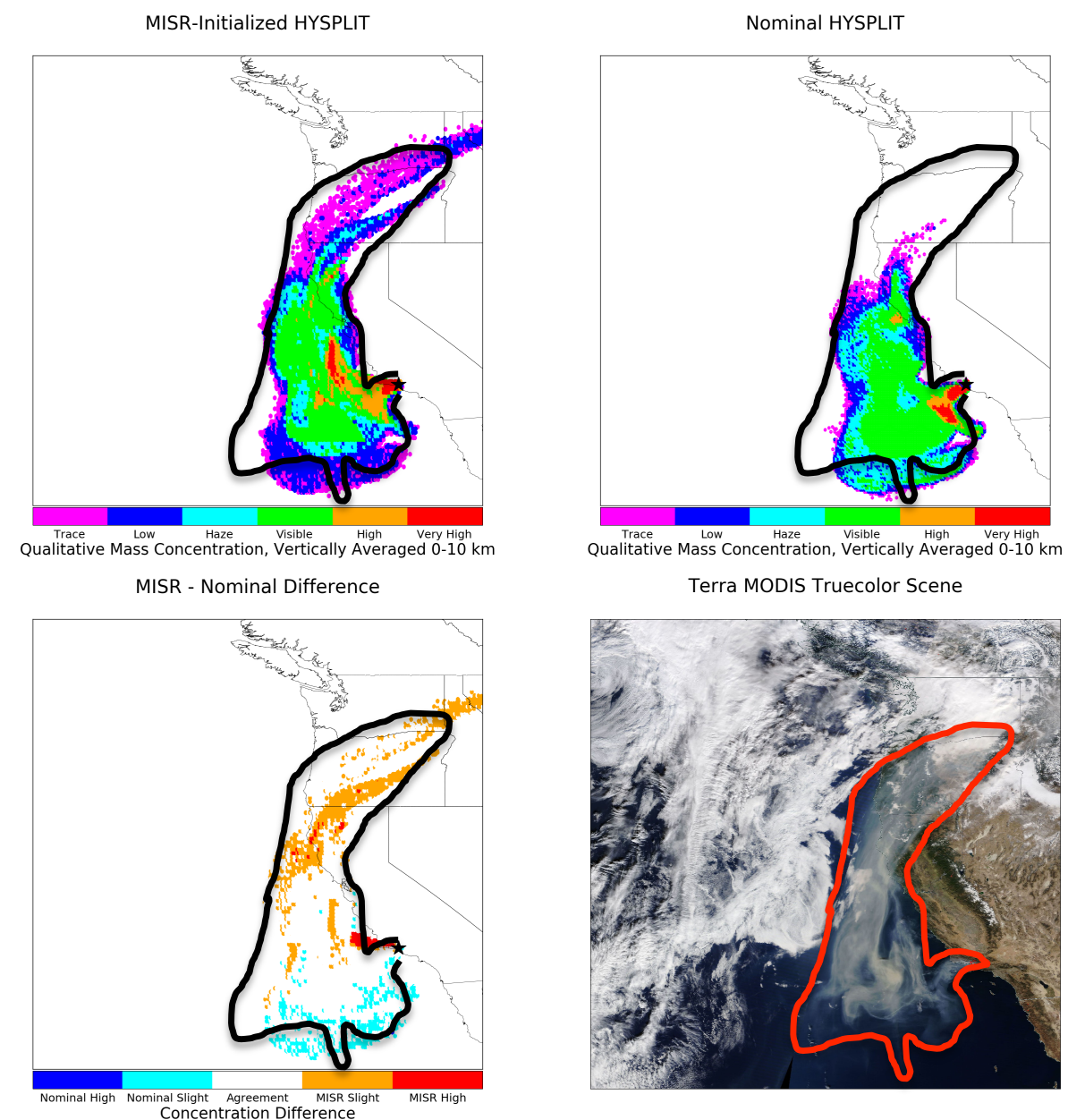

1

3 Eyjafjallajokull

$4 \quad$ Figure S18 
Eyjafjallajokull Eruption Simulation - May 7, 2010 (12:35 UTC)

MISR-Initialized HYSPLIT

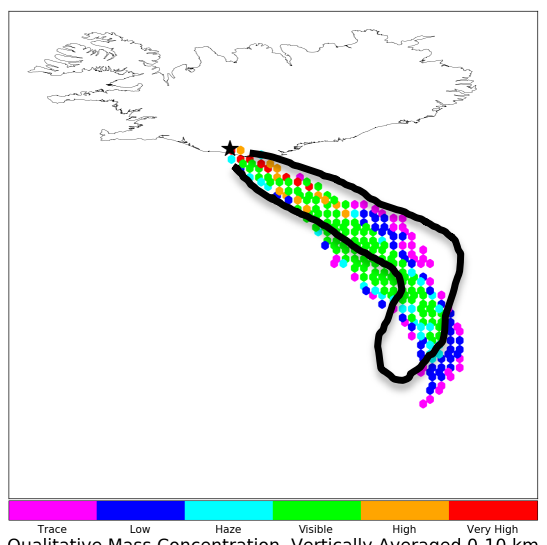

MISR - Nominal Difference

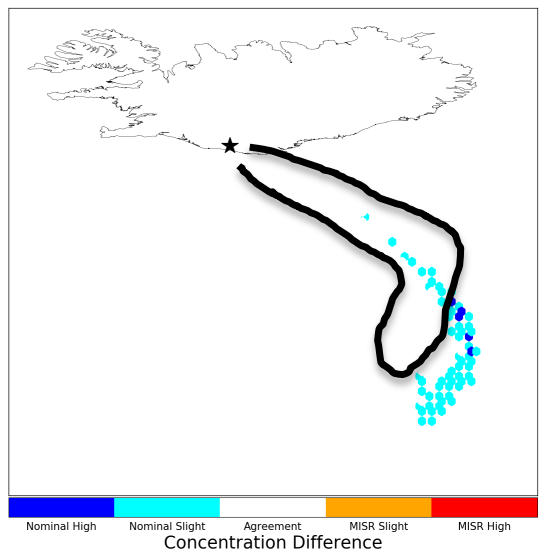

Nominal HYSPLIT

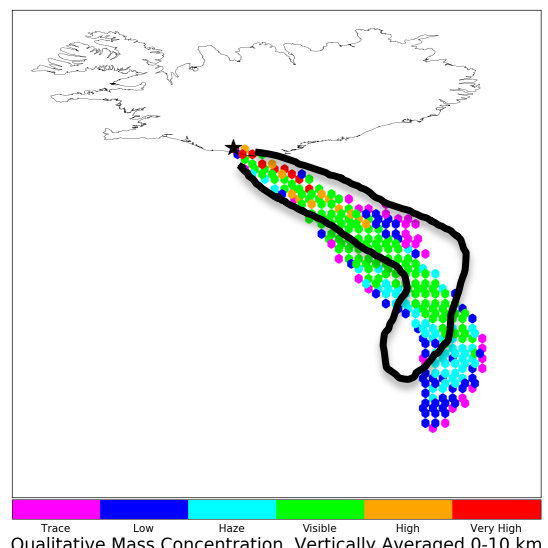

Terra MODIS Truecolor Scene

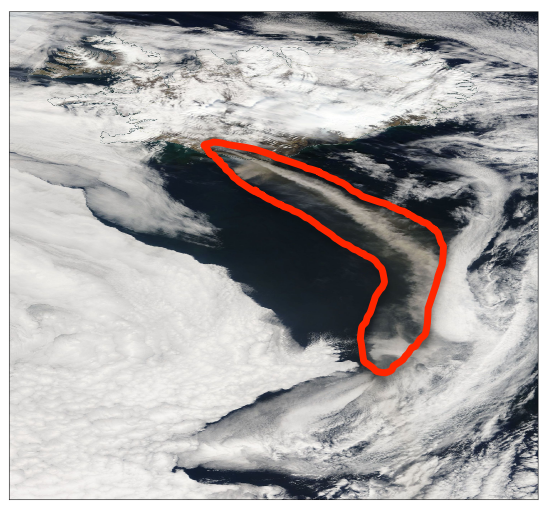


Eyjafjallajokull Eruption Simulation - May 8, 2010 (13:20 UTC)
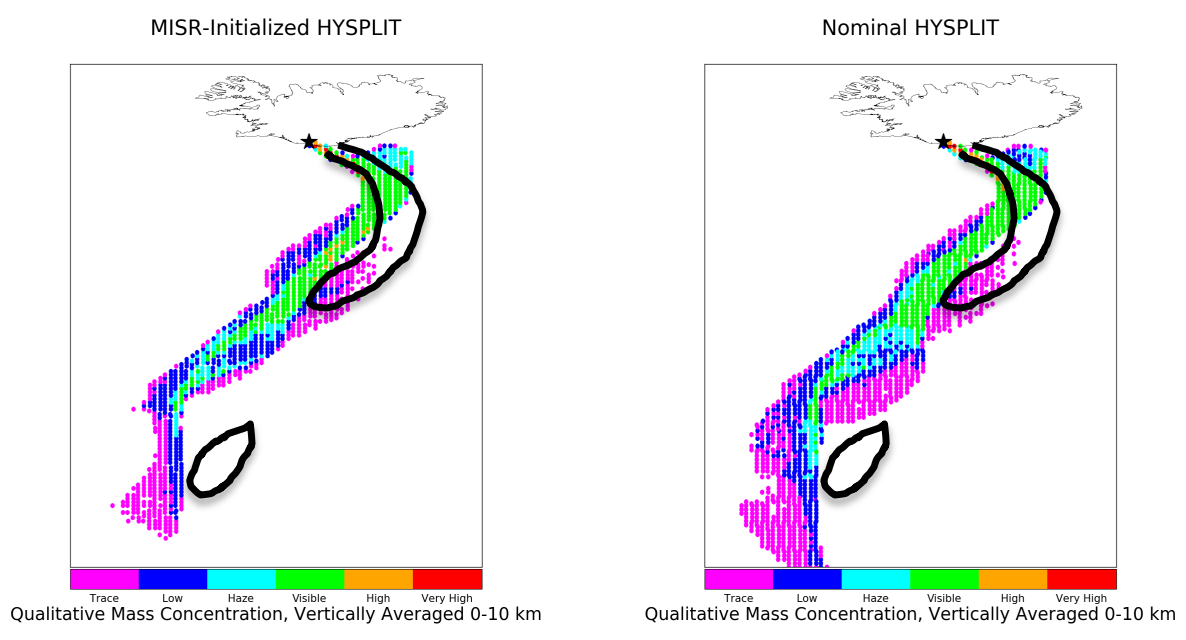

MISR - Nominal Difference

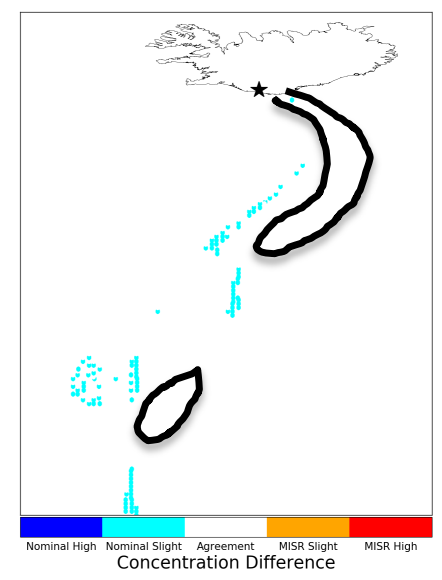

Terra MODIS Truecolor Scene

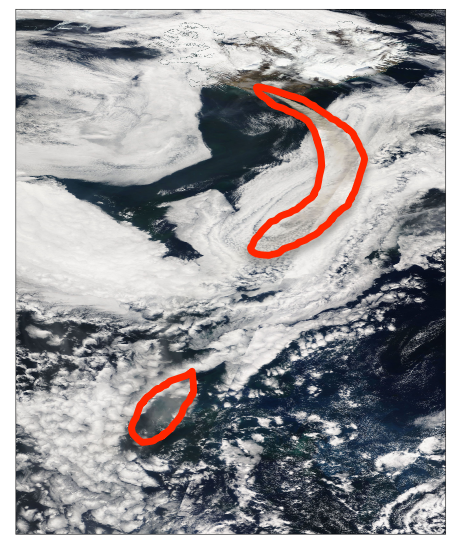


Eyjafjallajokull Eruption Simulation - May 10, 2010 (13:25 UTC)
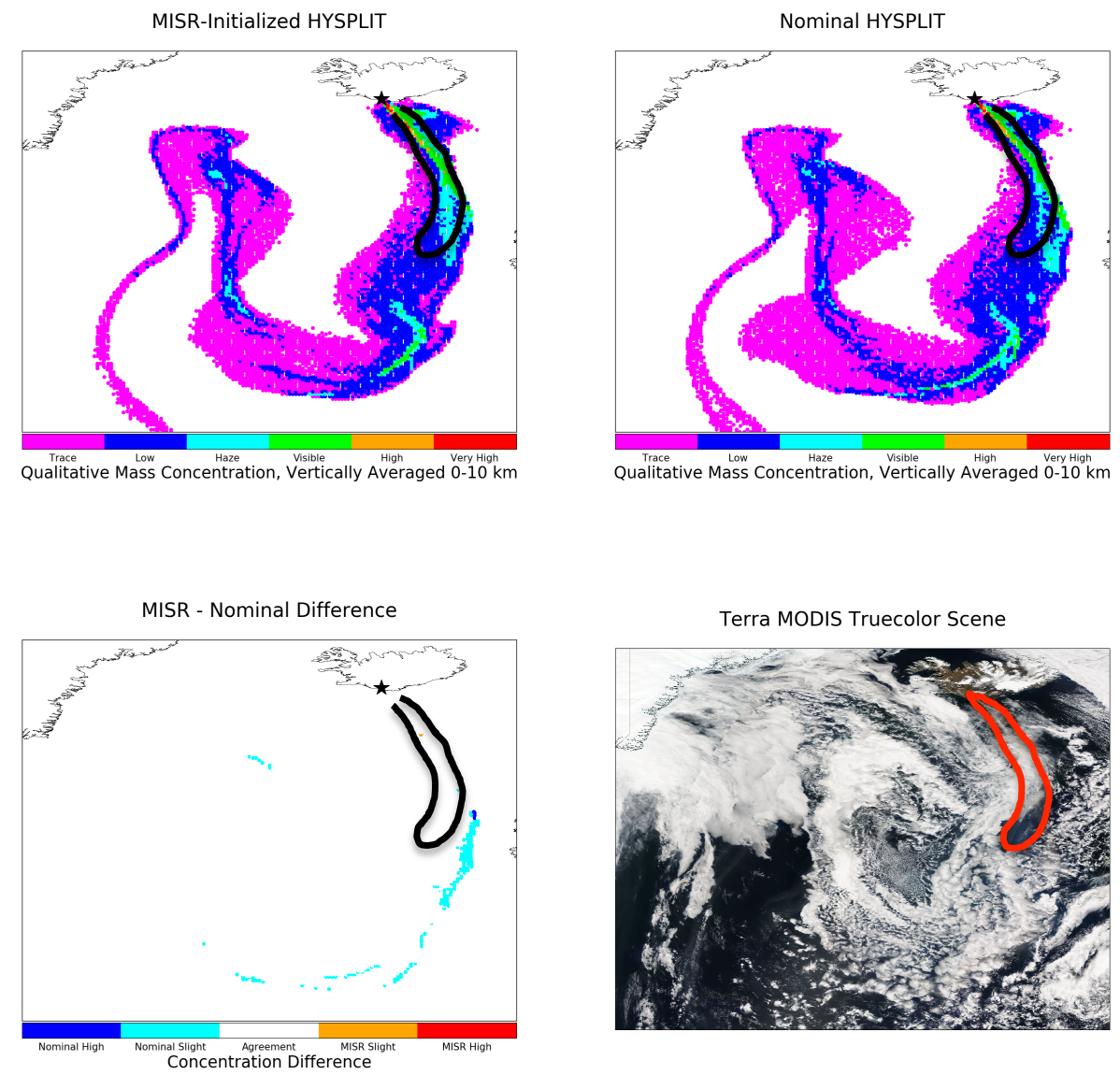

1

3 Etna

$4 \quad$ Figure S21 
Mount Etna Eruption Simulation - July 23, 2001 (10:35 UTC)
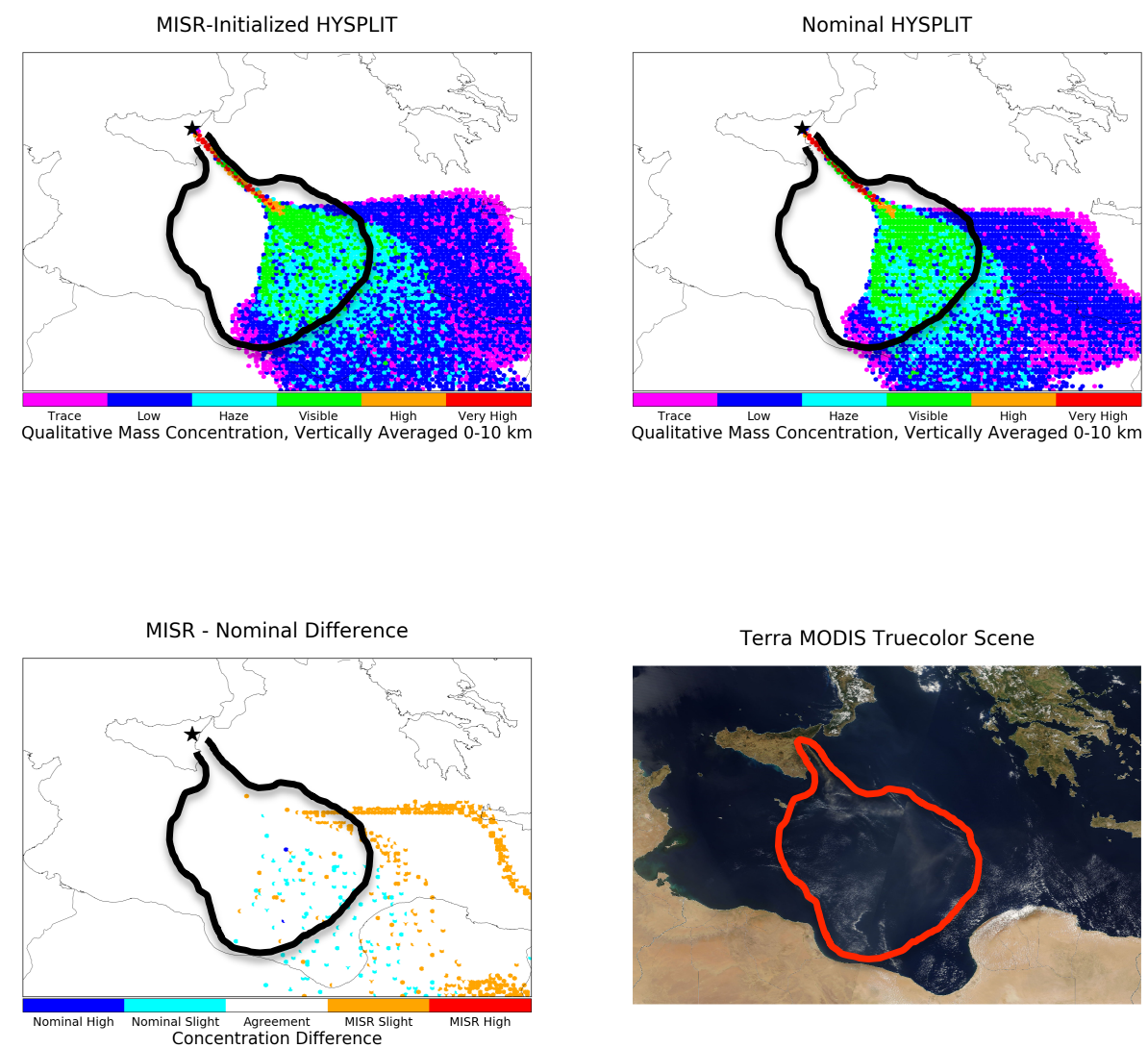
Mount Etna Eruption Simulation - July 24, 2001 (09:40 UTC)
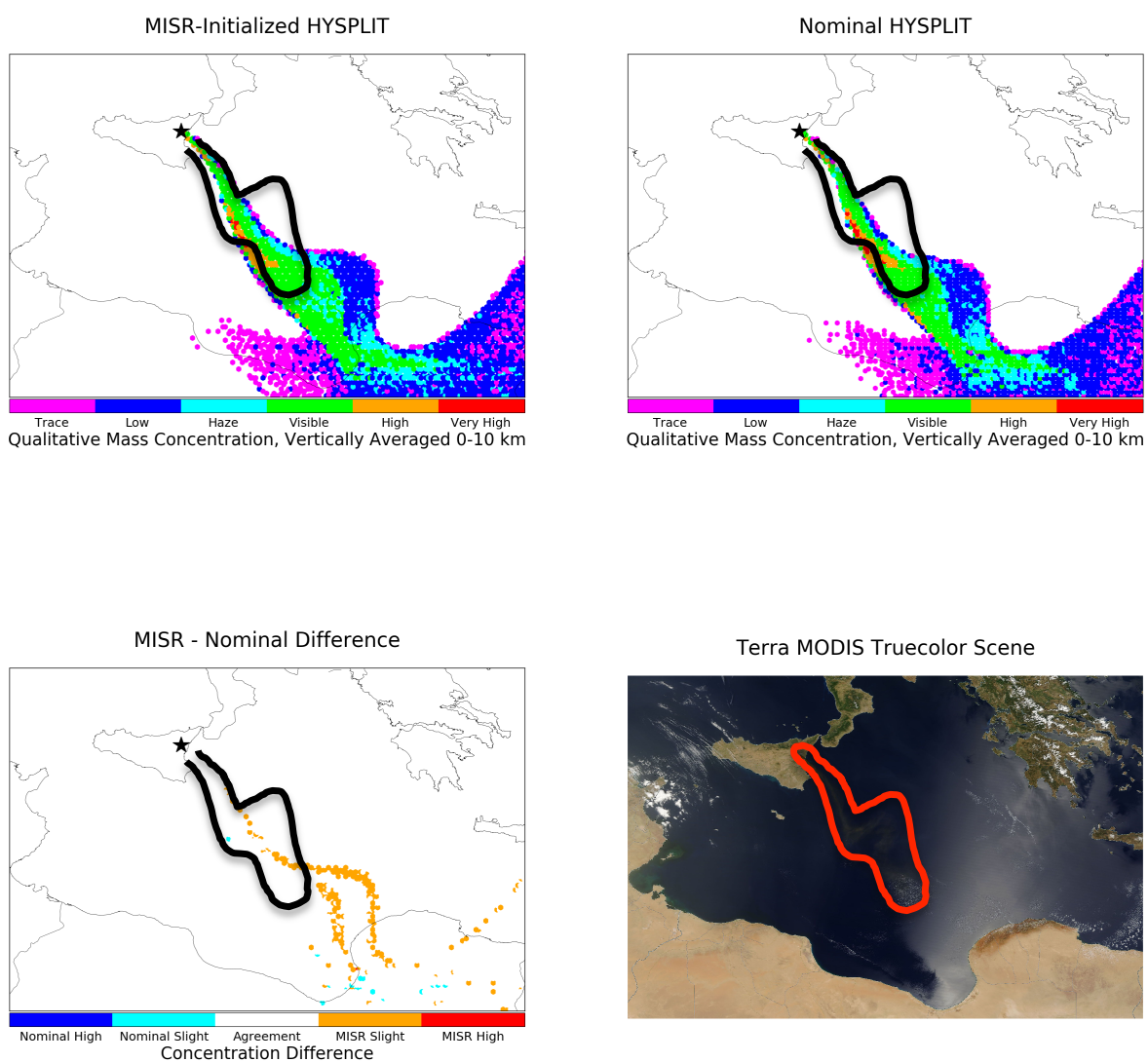
Mount Etna Eruption Simulation - July 25, 2001 (10:25 UTC)
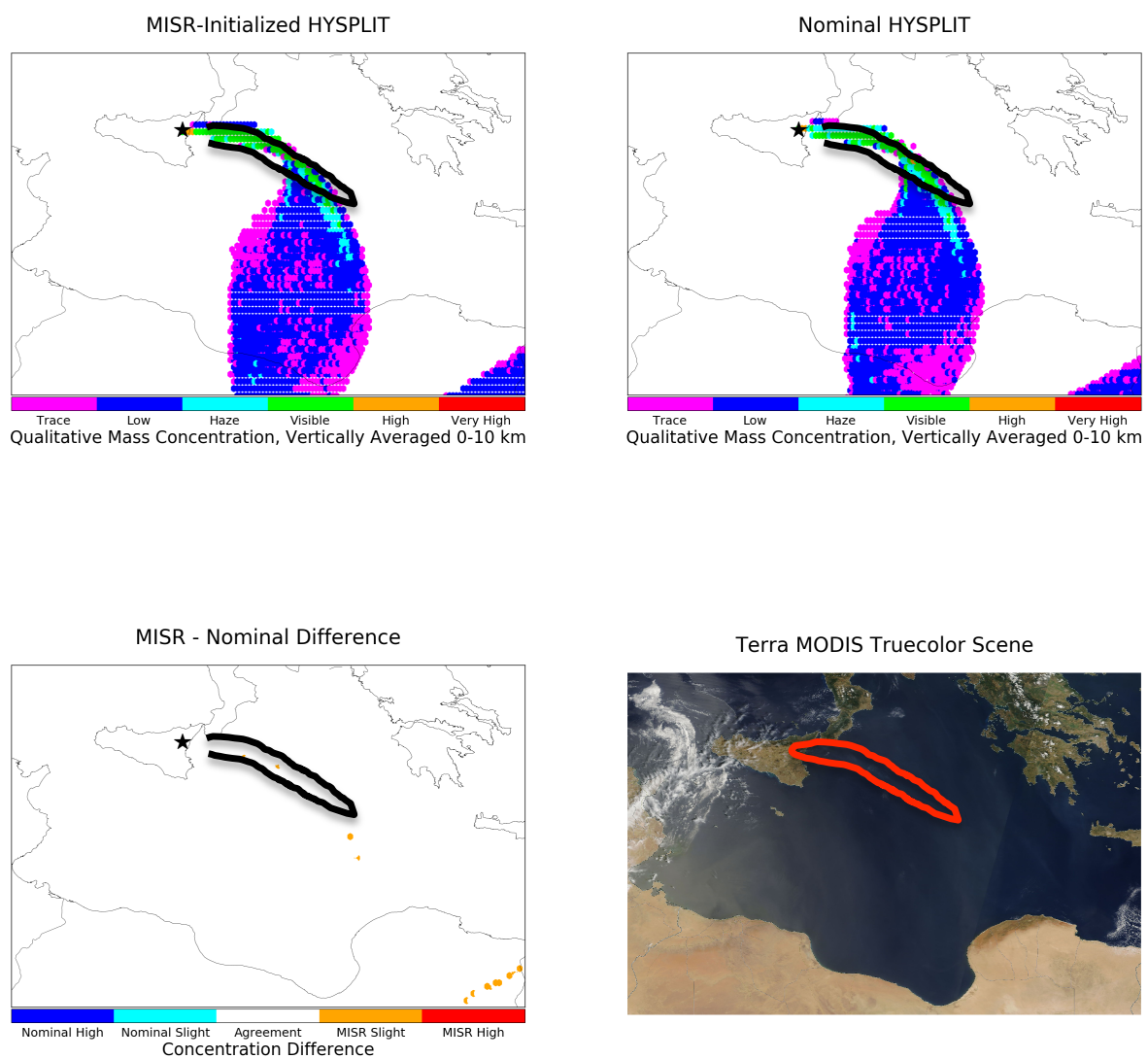

1

3 Chikurachki

$4 \quad$ Figure S24 
Chikurachki Eruption Simulation - April 22, 2003 (00:45 UTC)

MISR-Initialized HYSPLIT

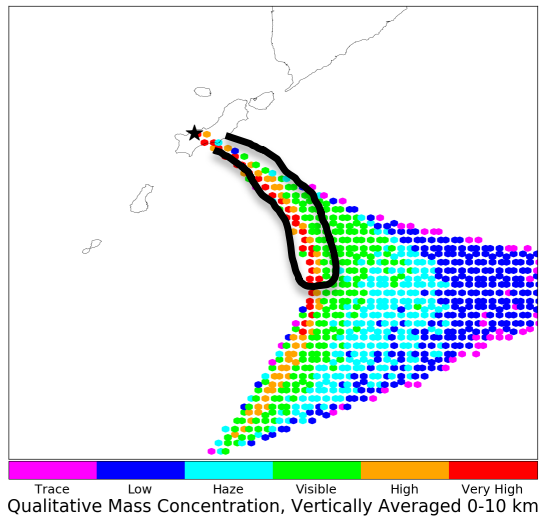

MISR - Nominal Difference

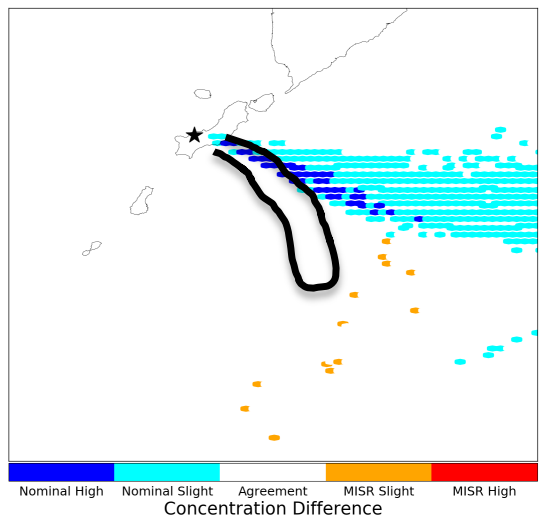

Nominal HYSPLIT

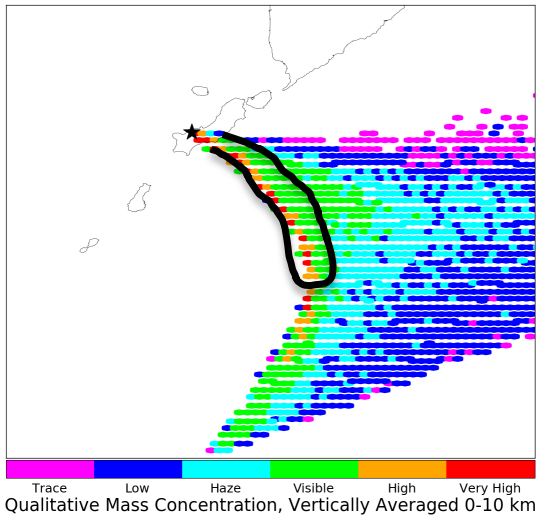

Terra MODIS Truecolor Scene

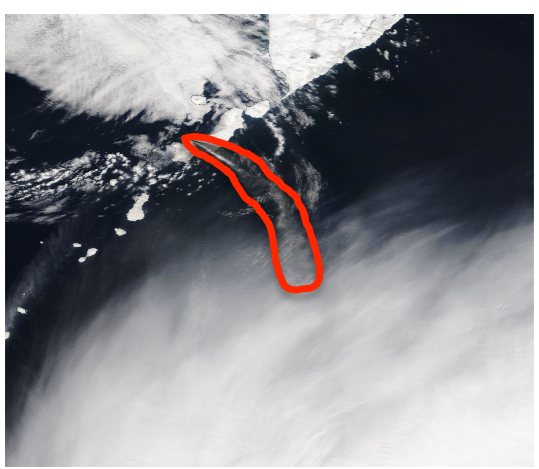


Chikurachki Eruption Simulation - April 23, 2003 (01:25 UTC)

MISR-Initialized HYSPLIT

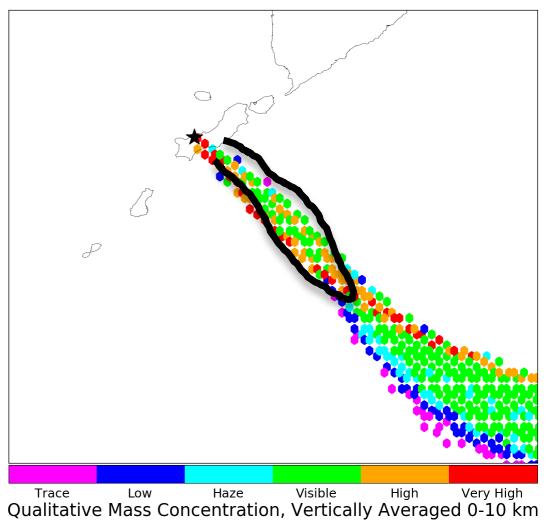

MISR - Nominal Difference

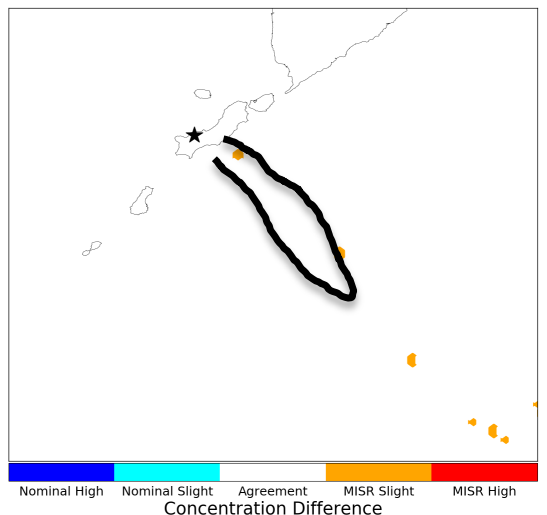

Nominal HYSPLIT

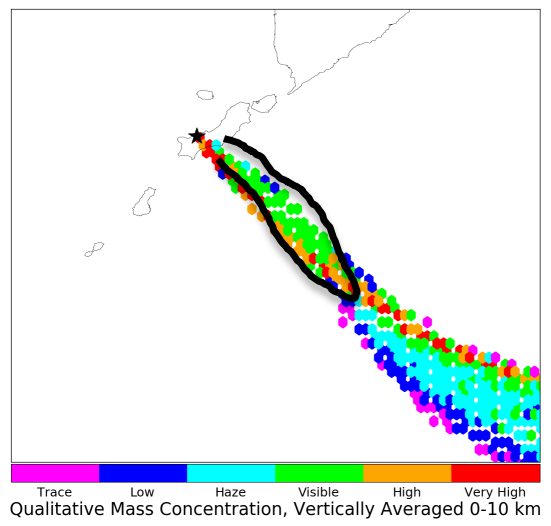

Terra MODIS Truecolor Scene

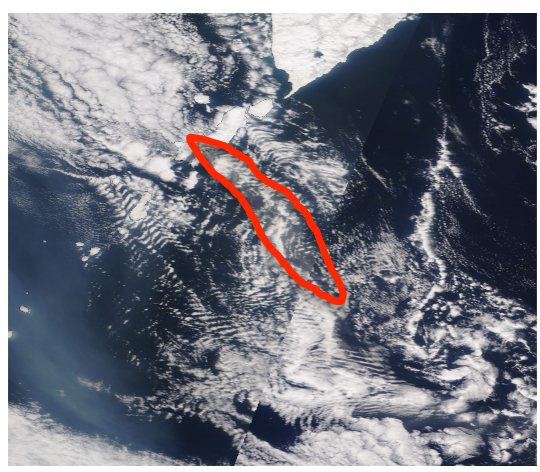


Chikurachki Eruption Simulation - April 24, 2003 (00:30 UTC)

MISR-Initialized HYSPLIT

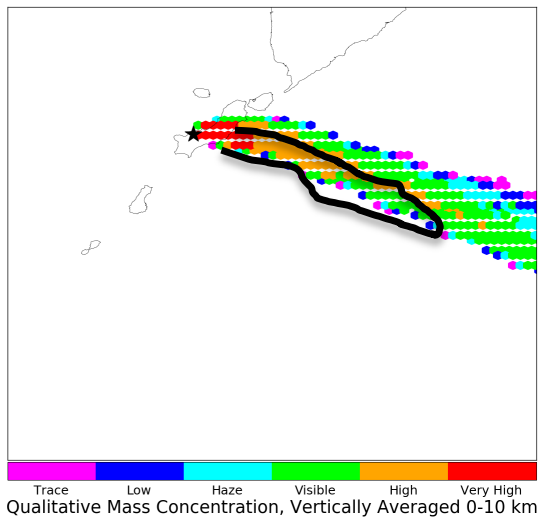

MISR - Nominal Difference

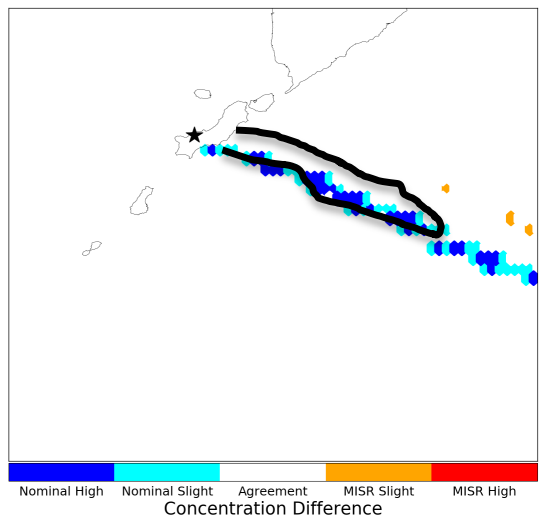

Nominal HYSPLIT

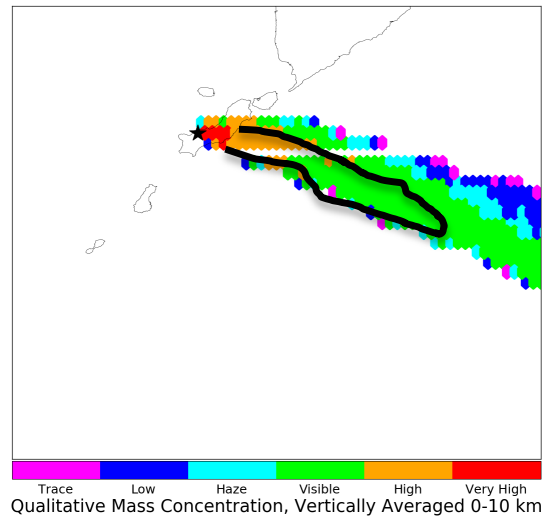

Terra MODIS Truecolor Scene

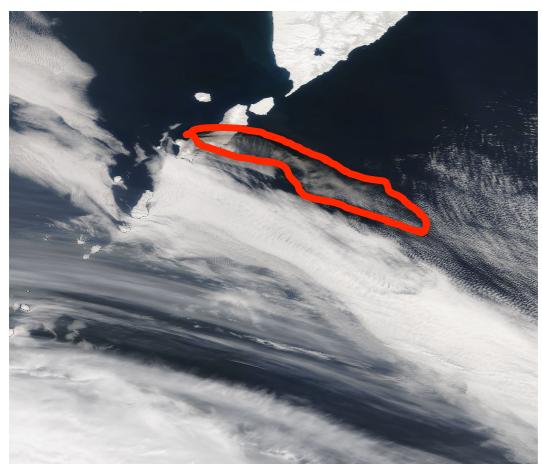

1

3 MINX Height and Wind Plots

4

5 Fort McMurray

6 Figure S27 

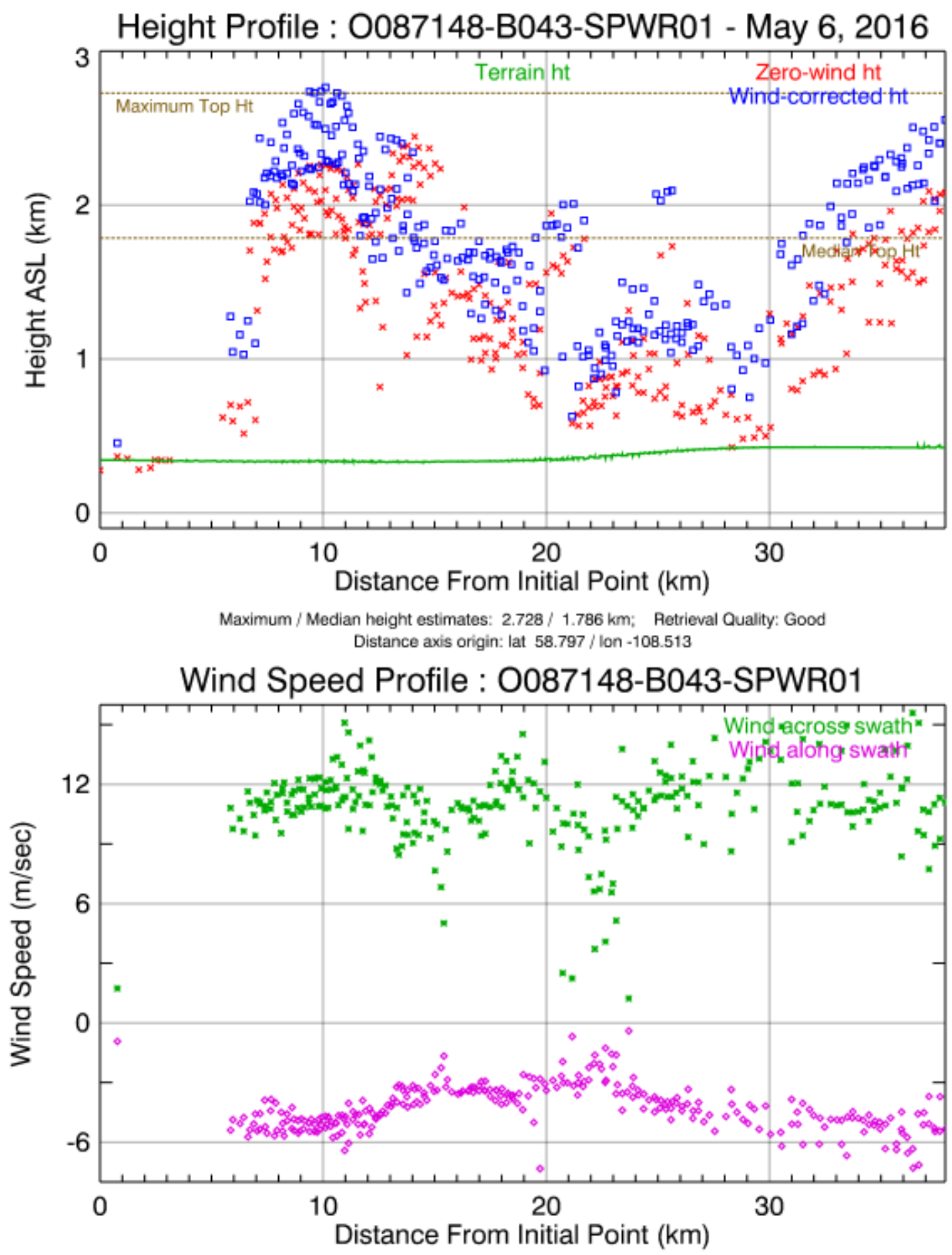

1

2 Figure $\mathbf{S 2 8}$ 

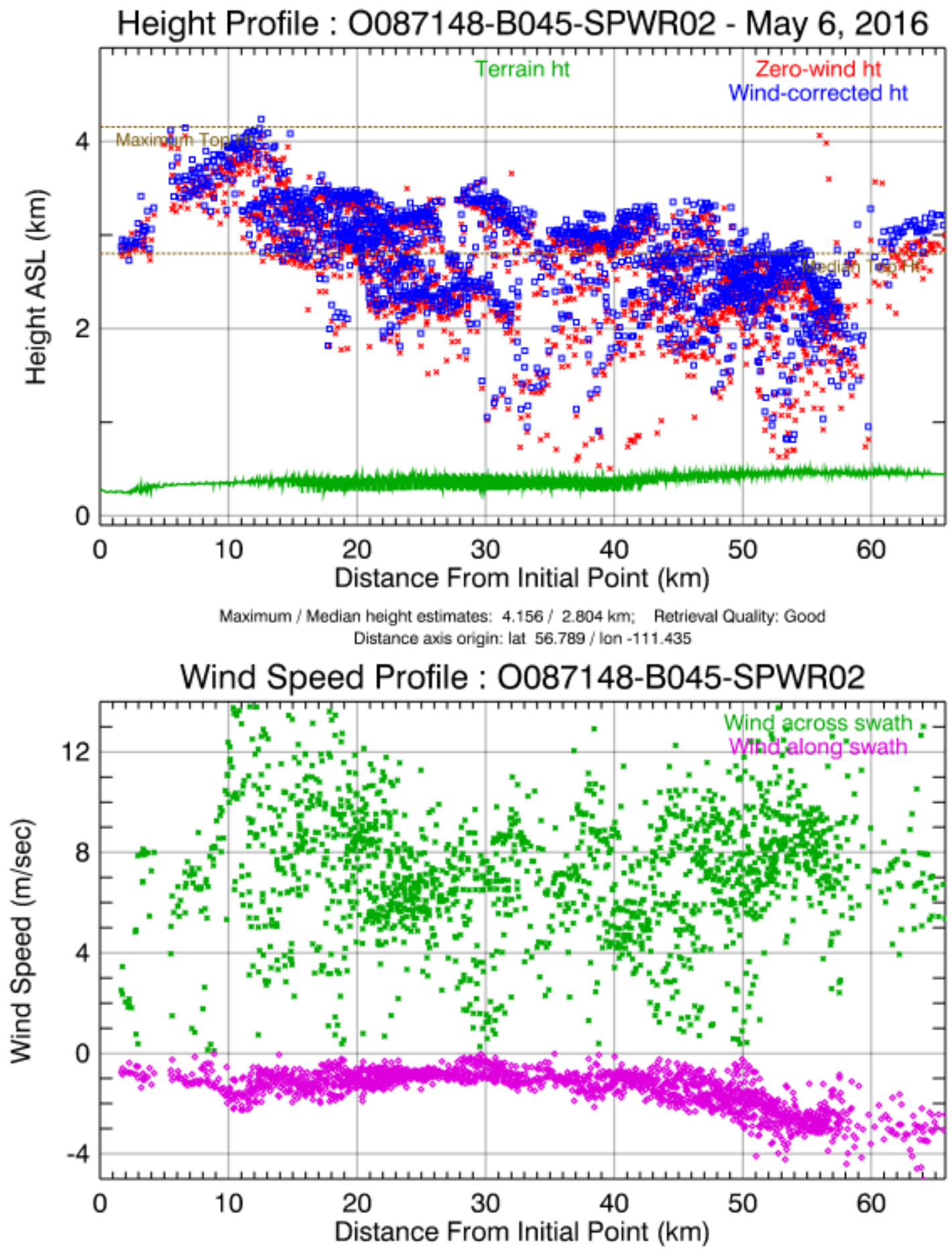


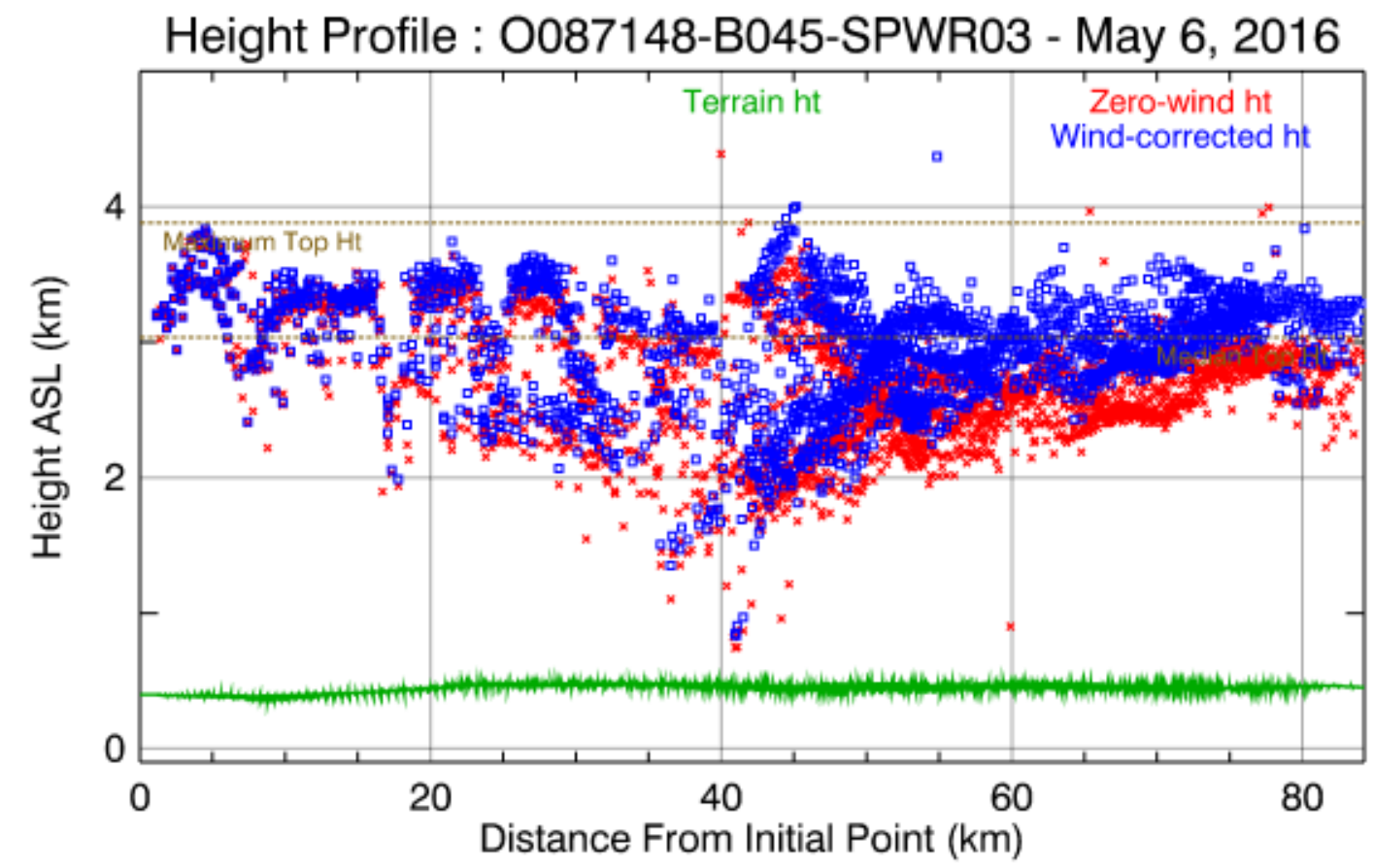

Maximum / Median height estimates: 3.880 / 3.036 km; Retrieval Quality: Good Distance axis origin: lat 56.581 / lon -111.540

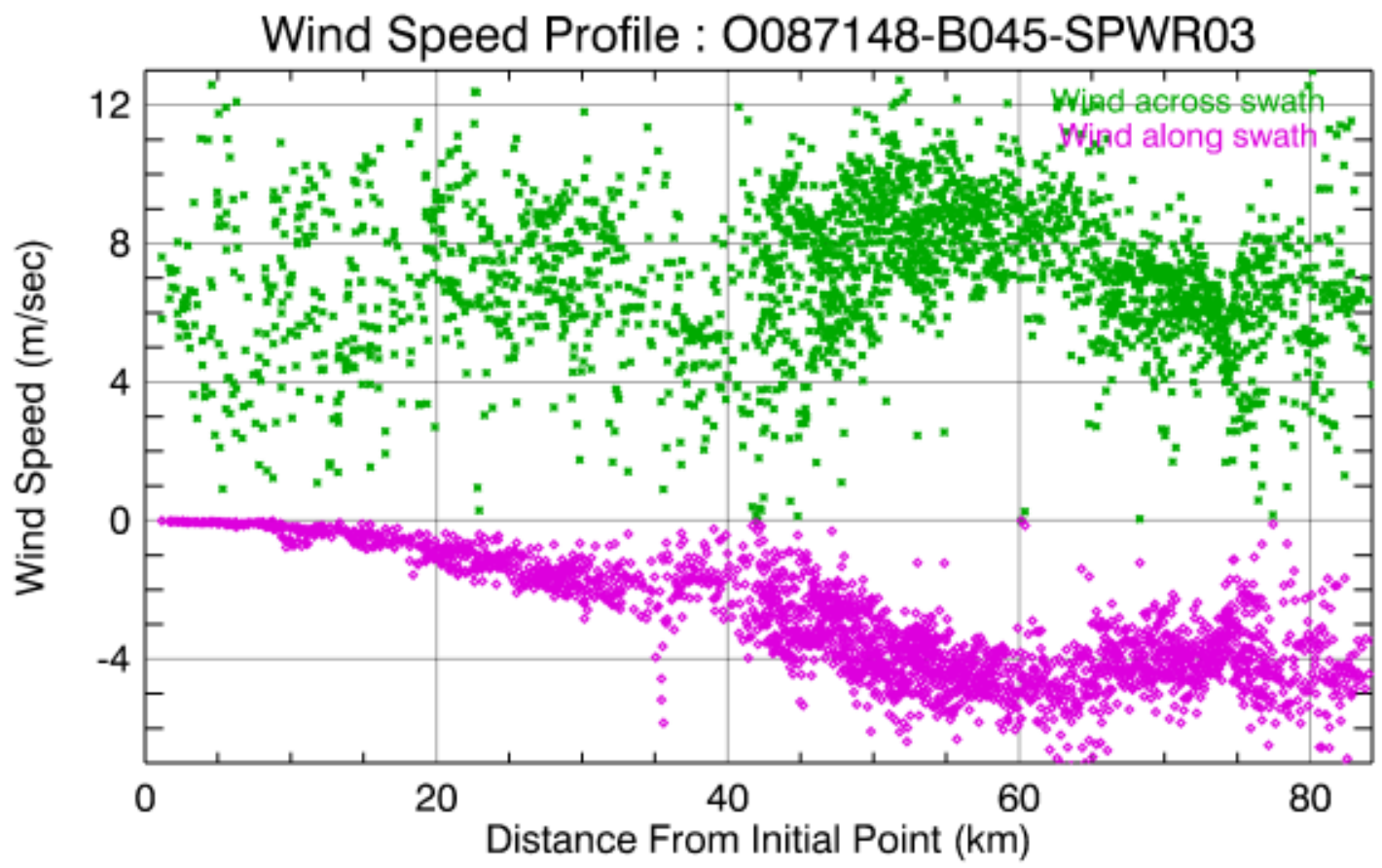


Height Profile : O087148-B045-SPWR04 - May 6, 2016

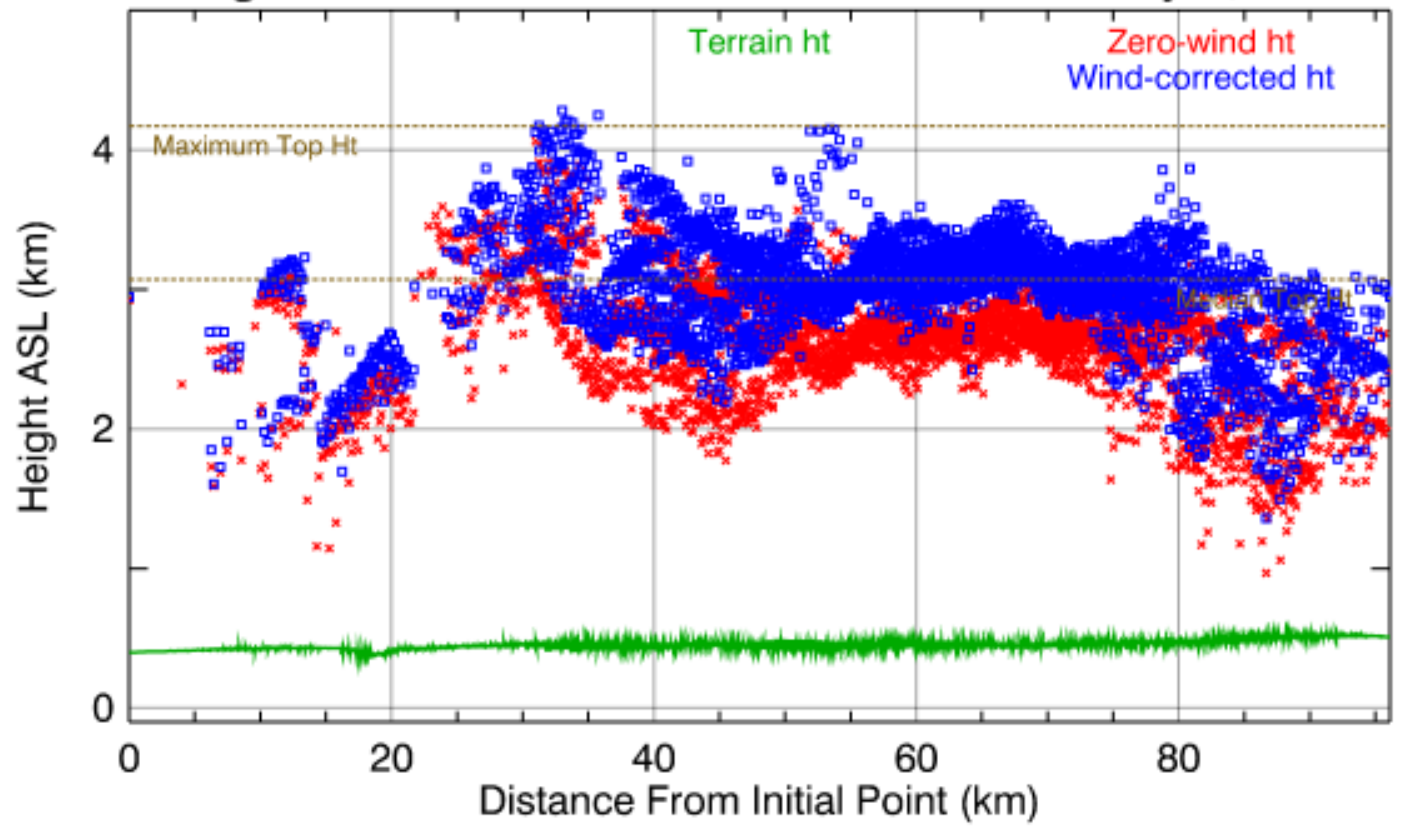

Maximum / Median height estimates: 4.169 / 3.070 km; Retrieval Quality: Good Distance axis origin: lat $56.590 /$ lon -111.060

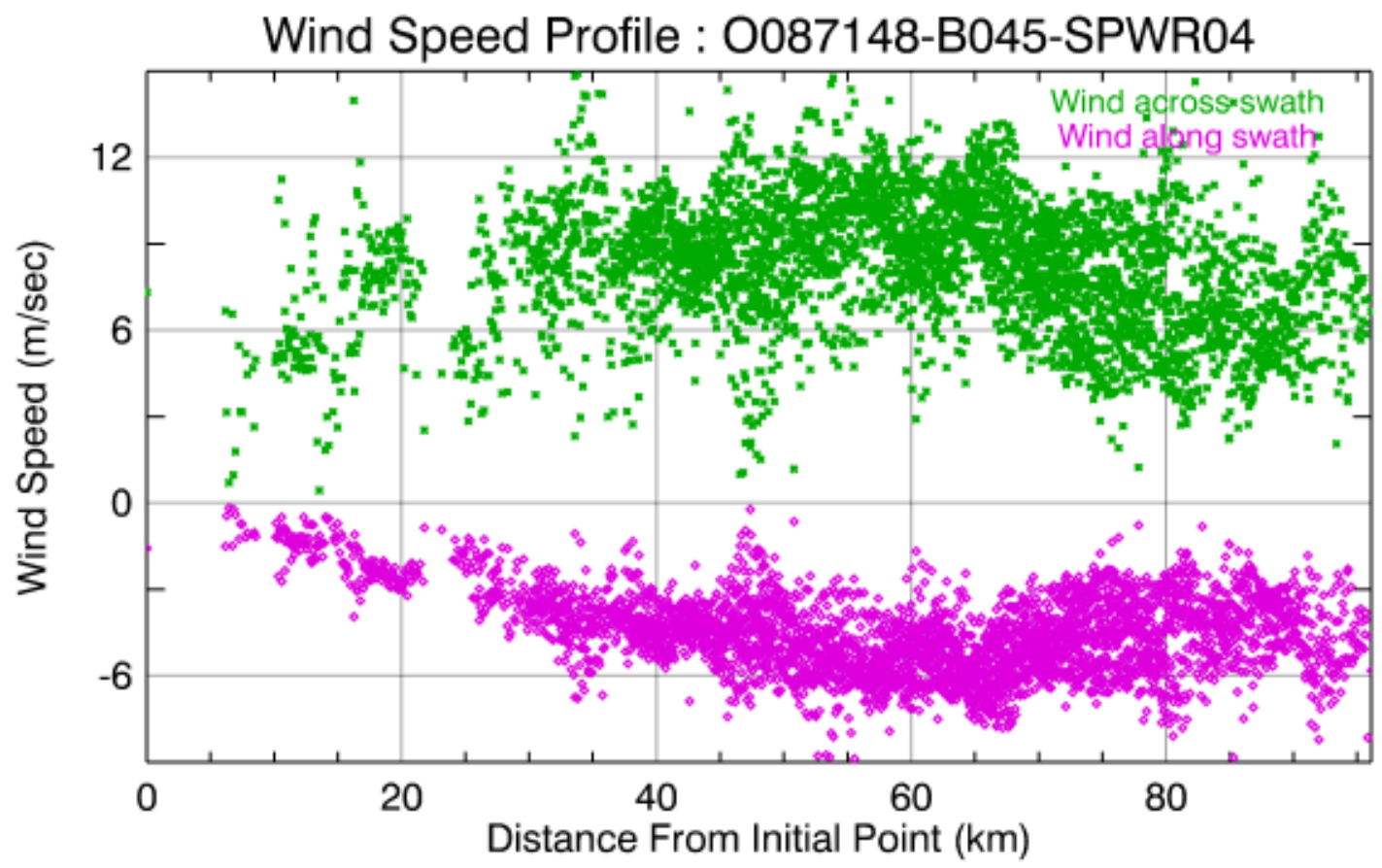

1

Fraser Plateau

$3 \quad$ Figure S31 

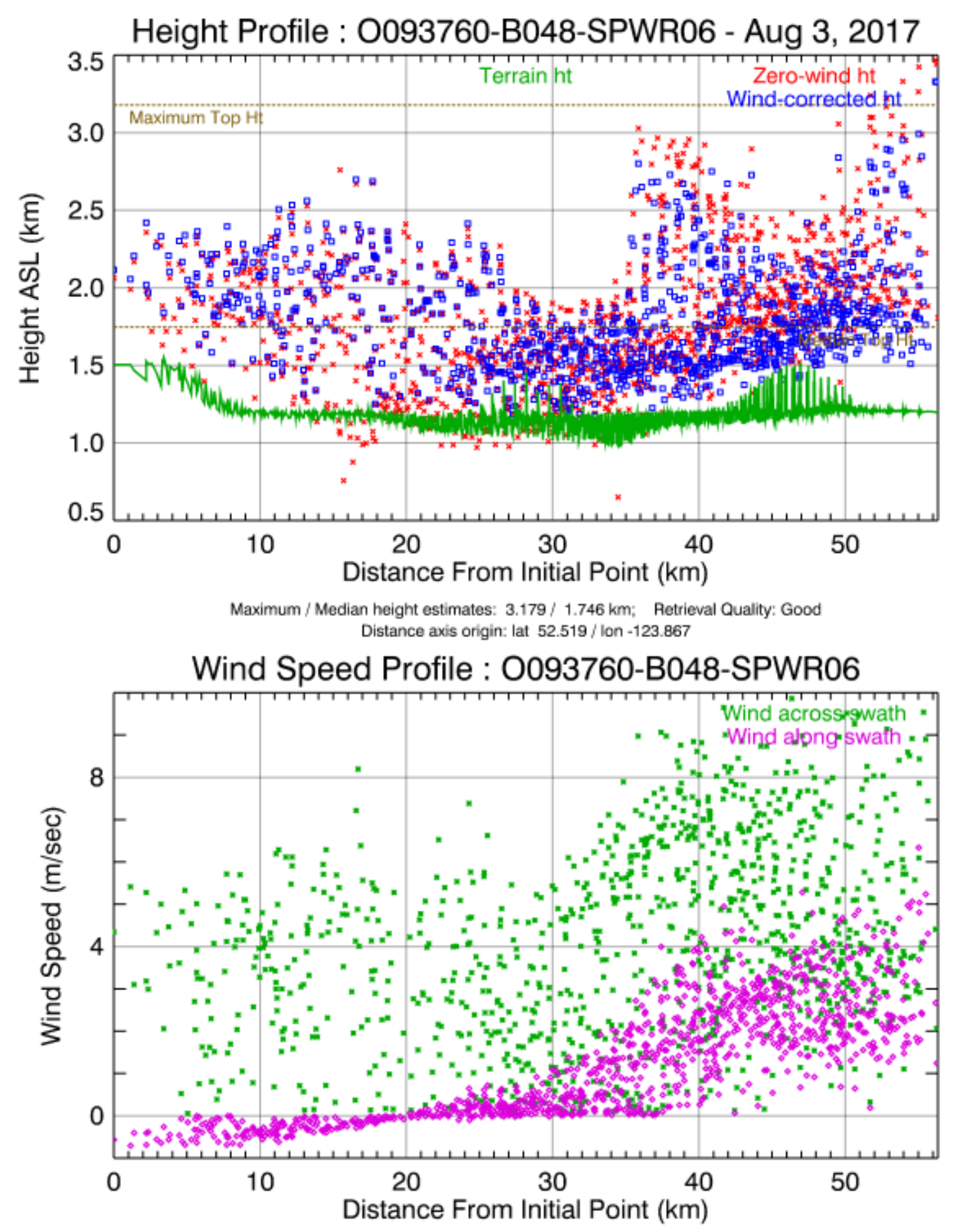
Height Profile : O093760-B048-SPWR05 - Aug 3, 2017

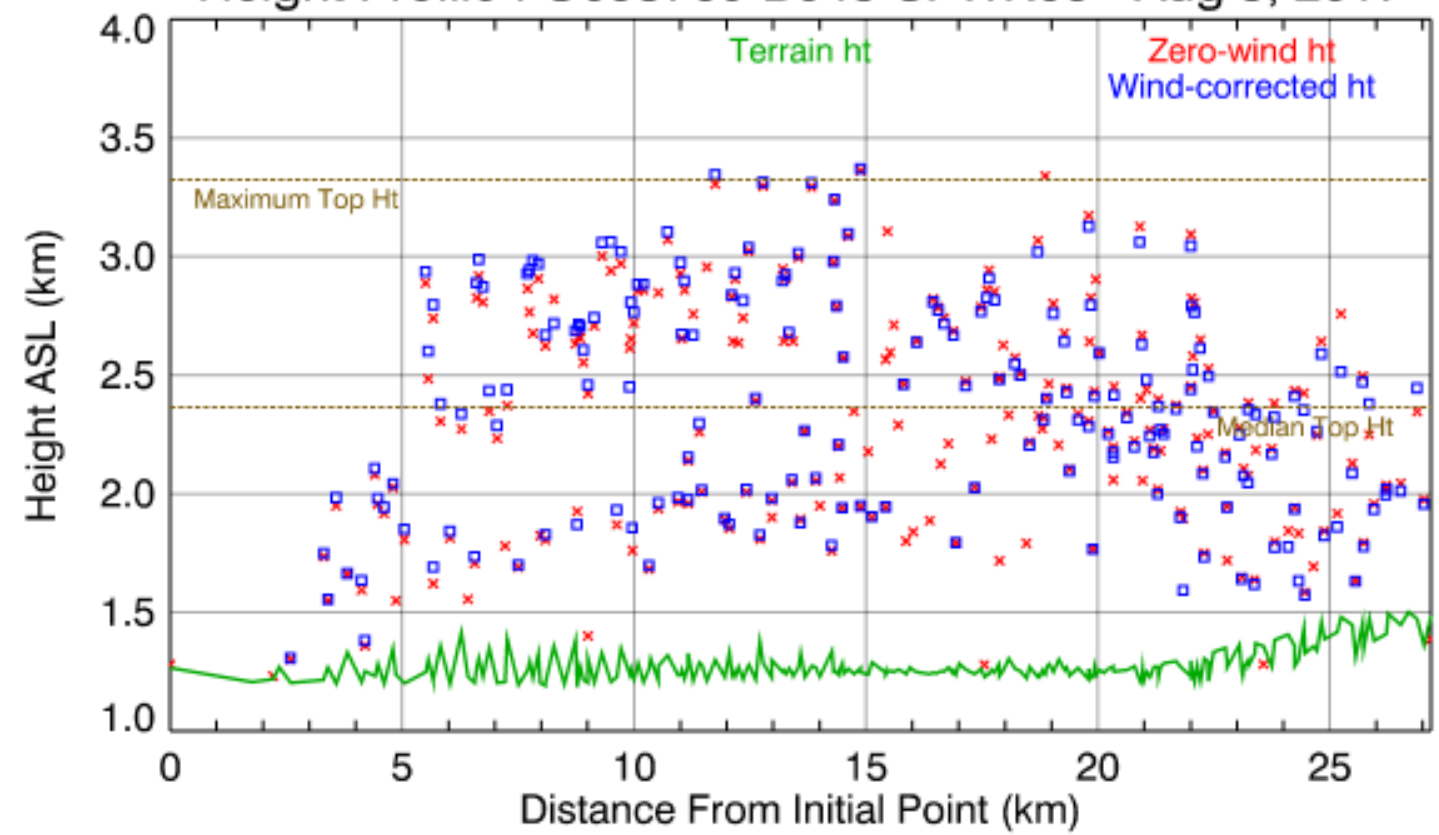

Maximum / Median height estimates: 3.324 / 2.364 km; Retrieval Quality: Good Distance axis origin: lat 52.678 / lon -124.216

Wind Speed Profile : O093760-B048-SPWR05

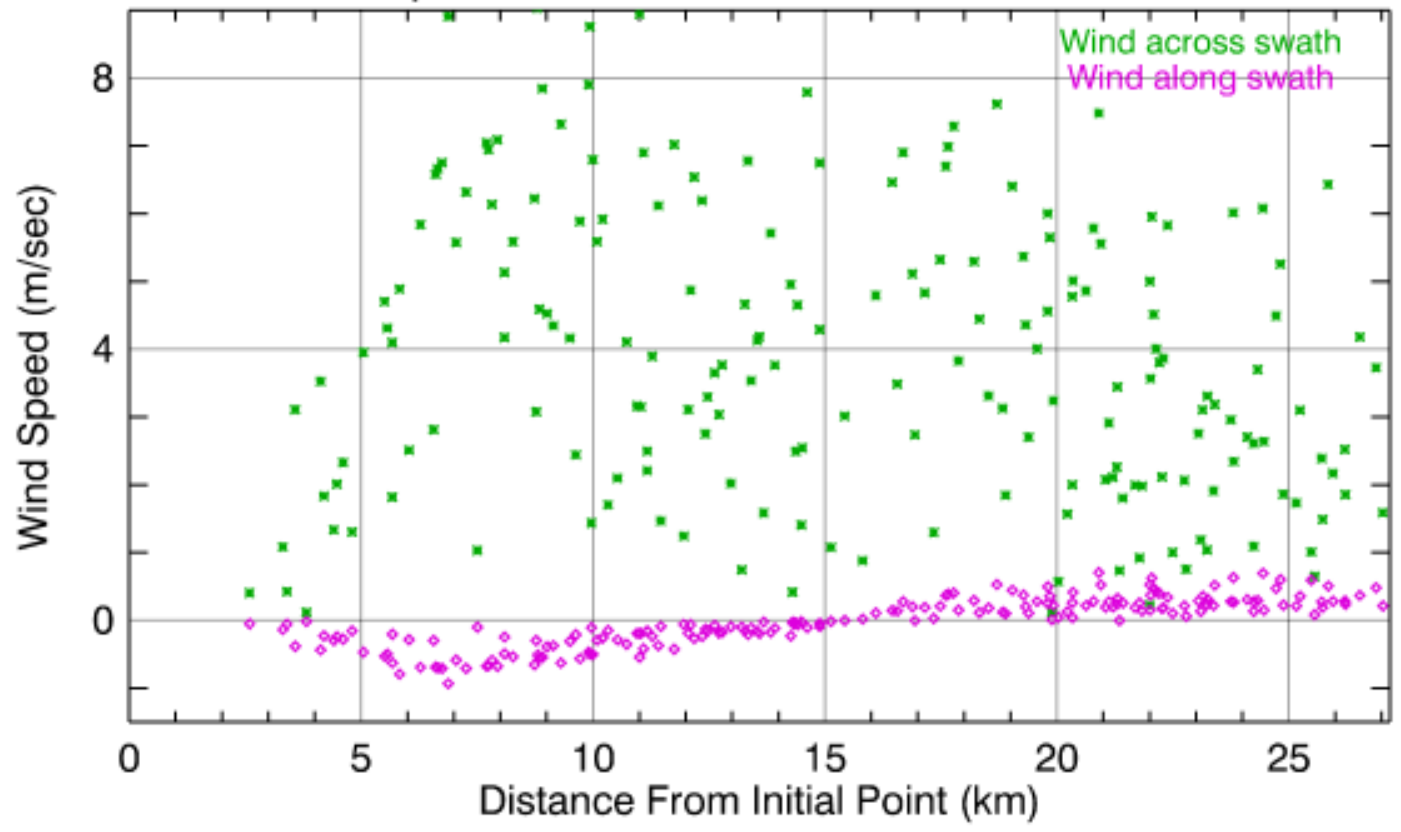



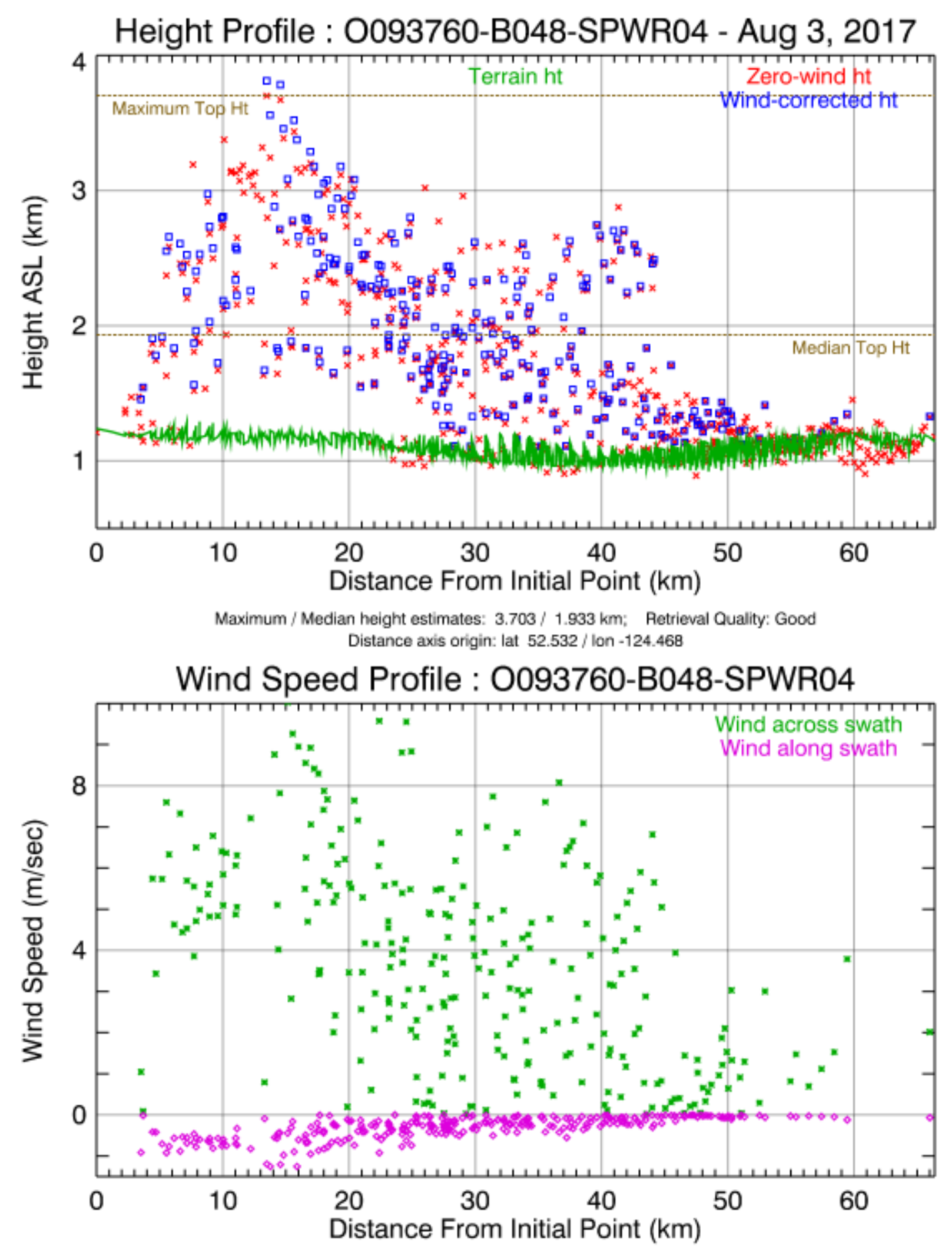

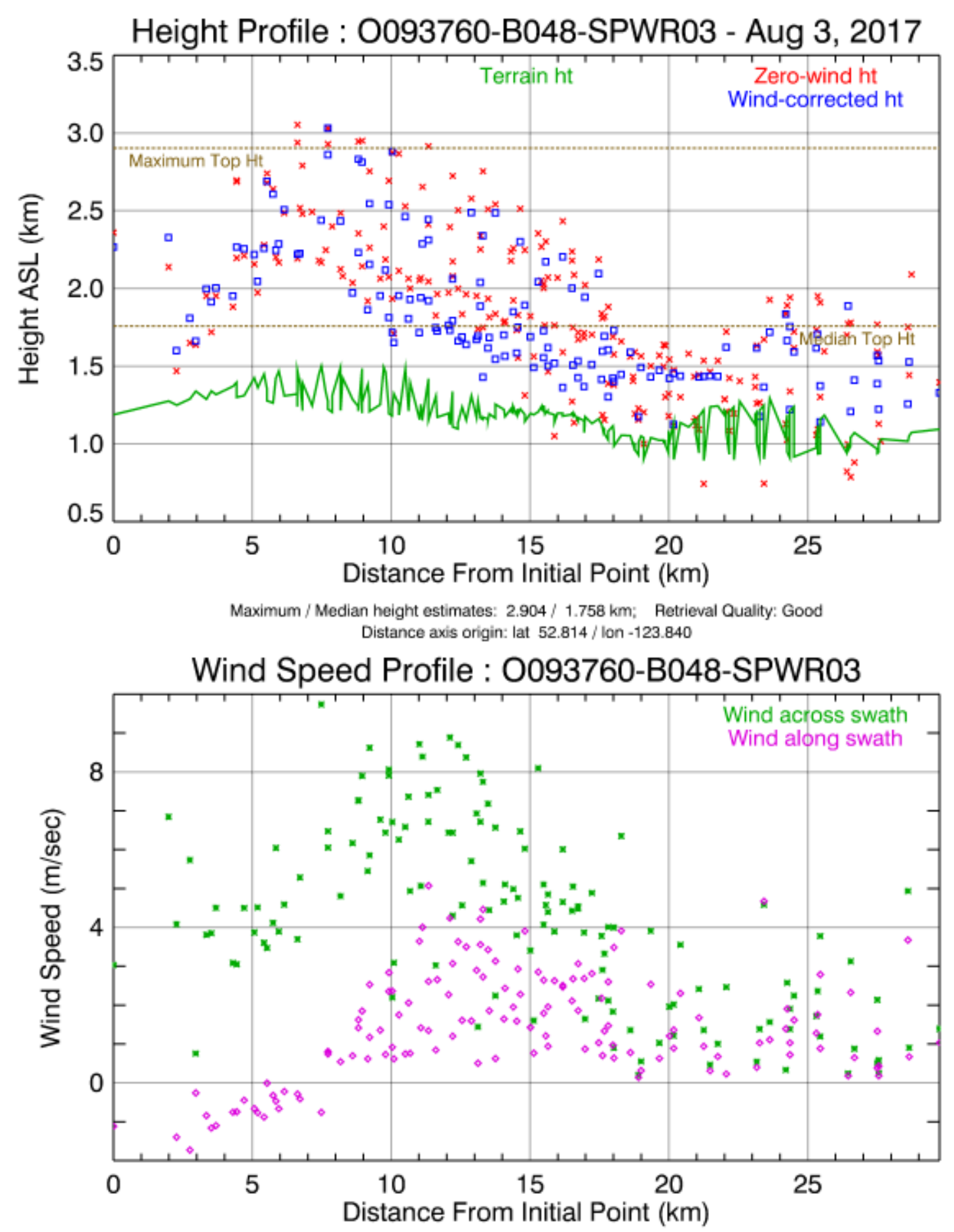

1

Figure S35 

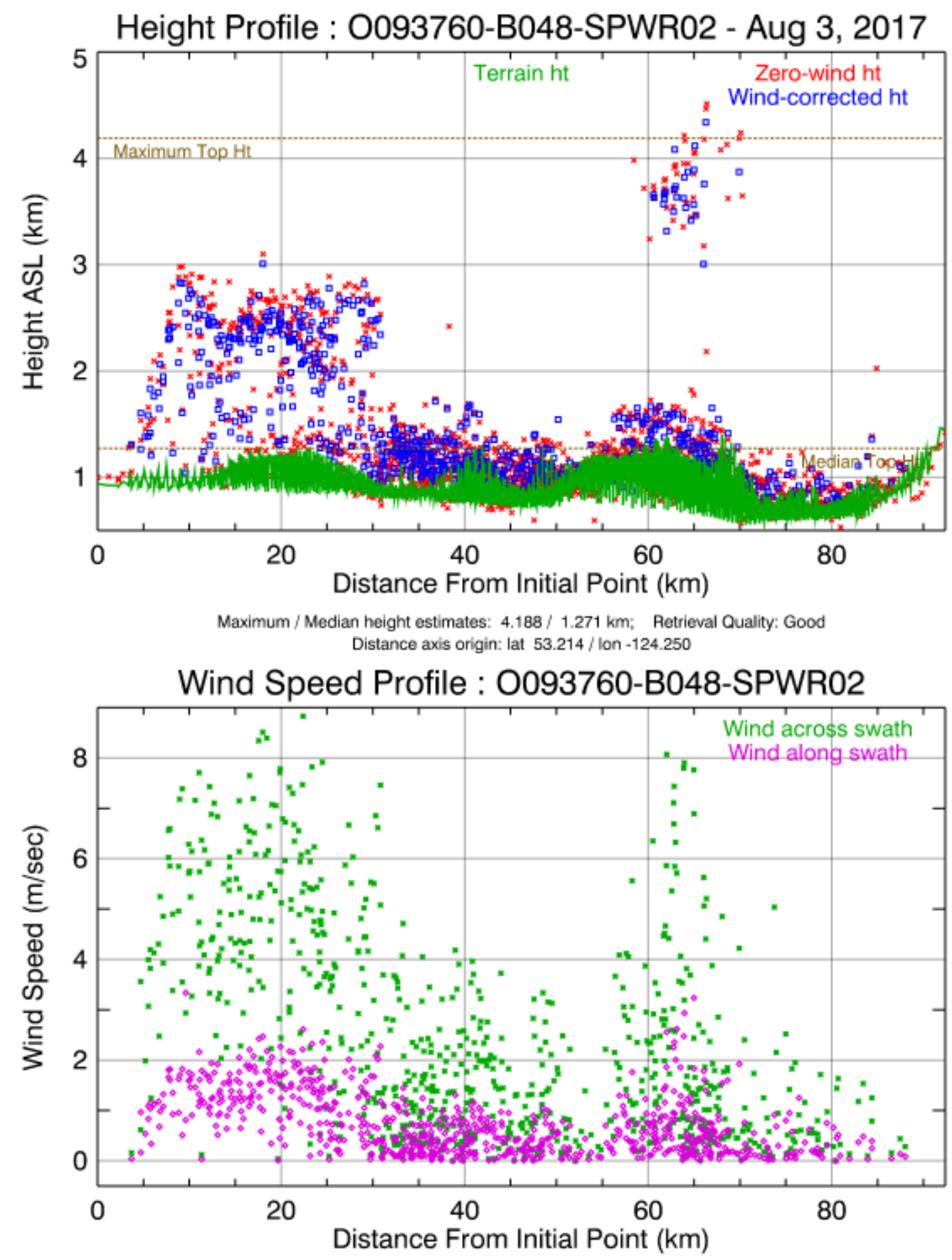

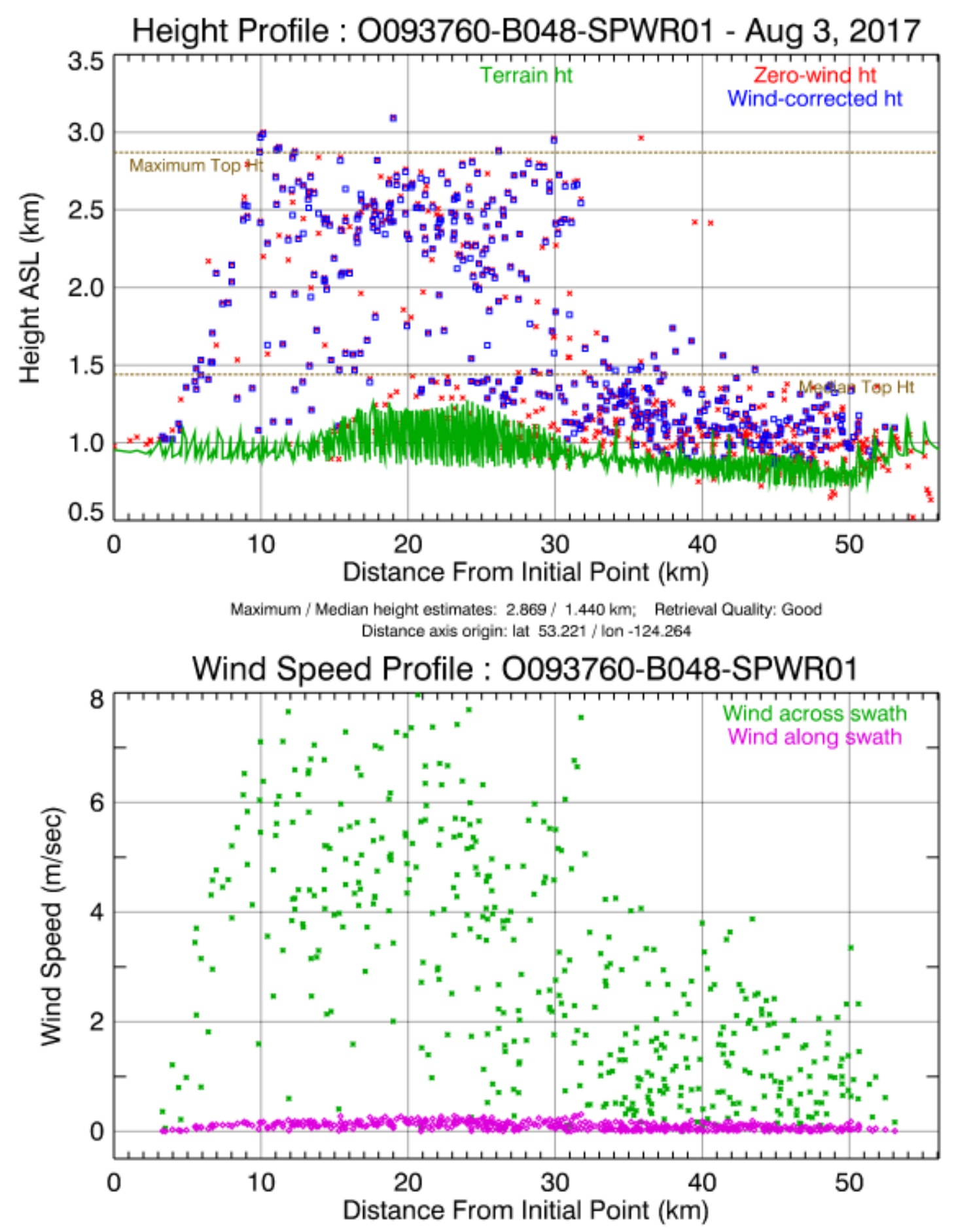
Height Profile : O095638-B063-SPWR02 - Dec 10, 2017

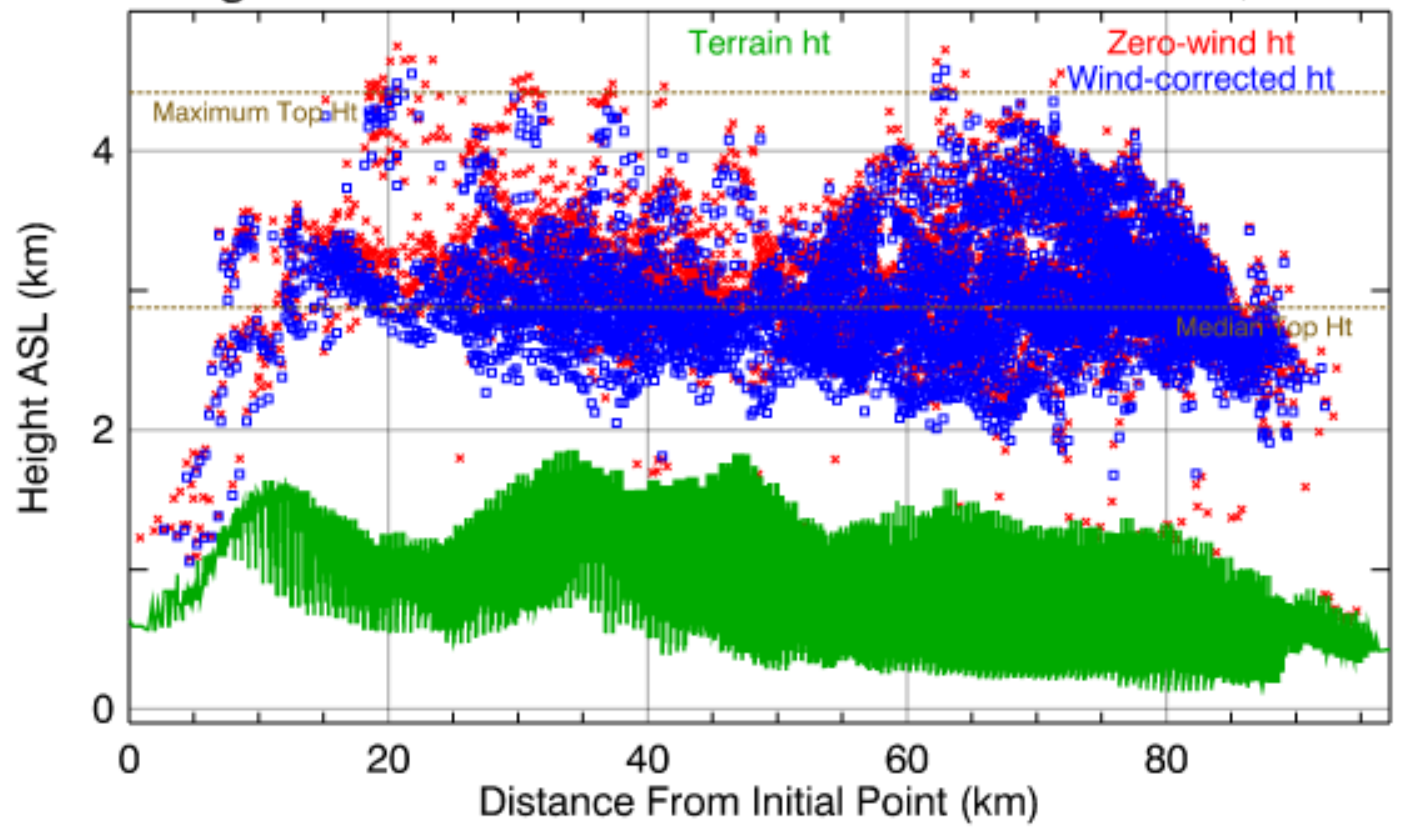

Maximum / Median height estimates: 4.417 / 2.878 km; Retrieval Quality: Good Distance axis origin: lat 34.506 / lon -119.358

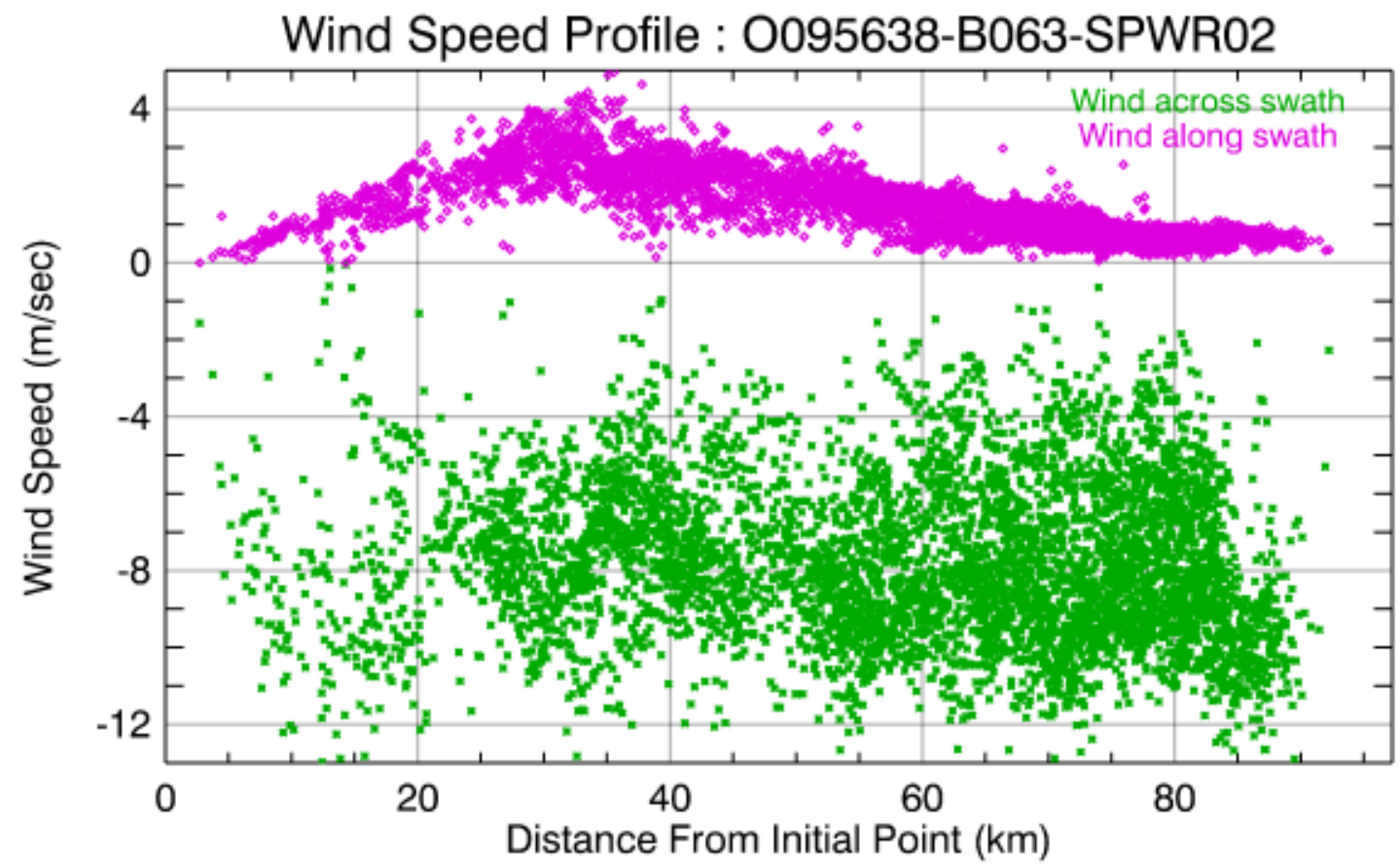


Height Profile : O095638-B063-SPWR01 - Dec 10, 2017

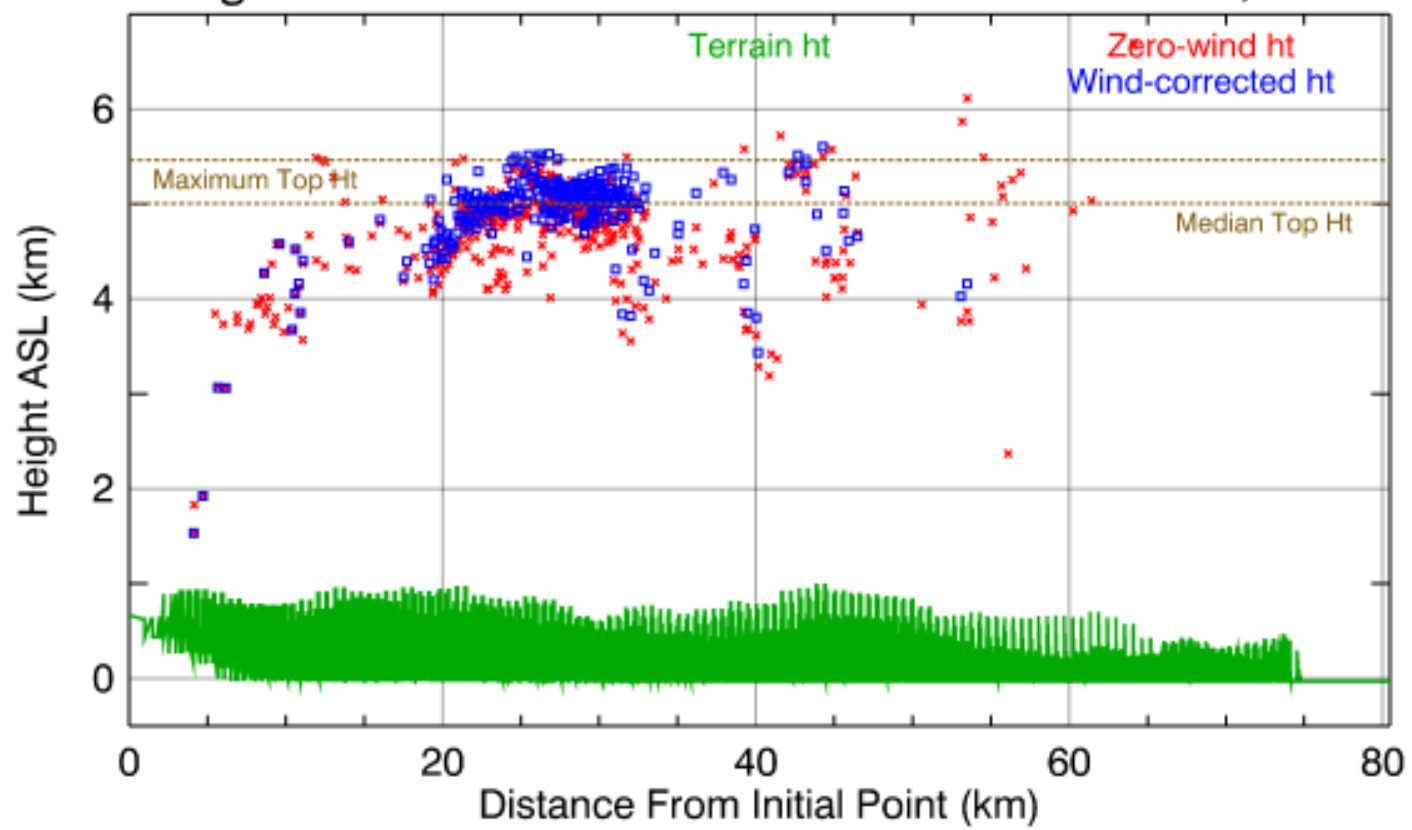

Maximum / Median height estimates: 5.461 / $5.006 \mathrm{~km}$; Retrieval Quality: Poor Distance axis origin: lat 34.450 / lon -119.504

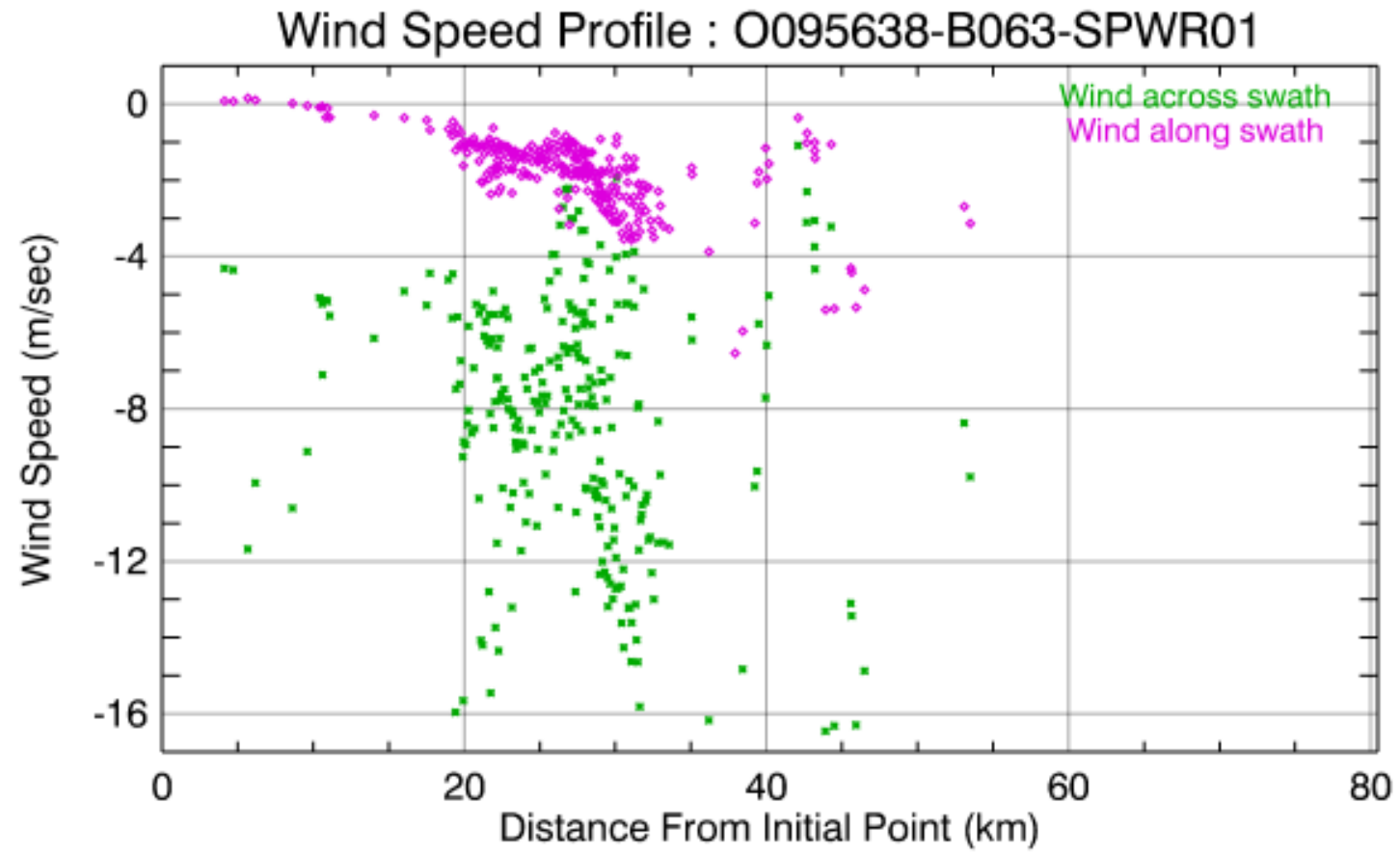

1

2

3 Eyjafjallajokull

$4 \quad$ Figure S39 

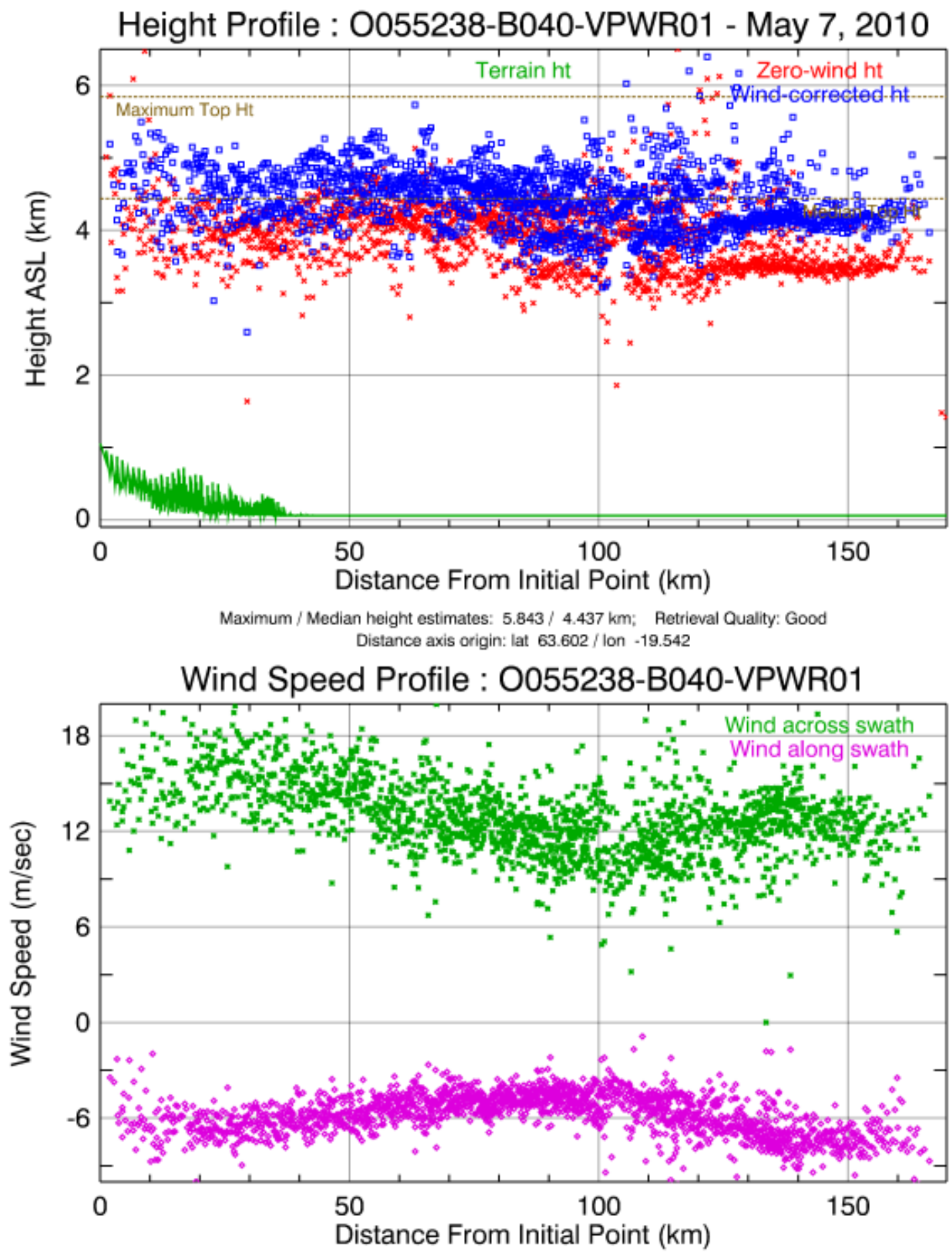
Height Profile : O008476-B060-VPWR01 - July 22, 2001

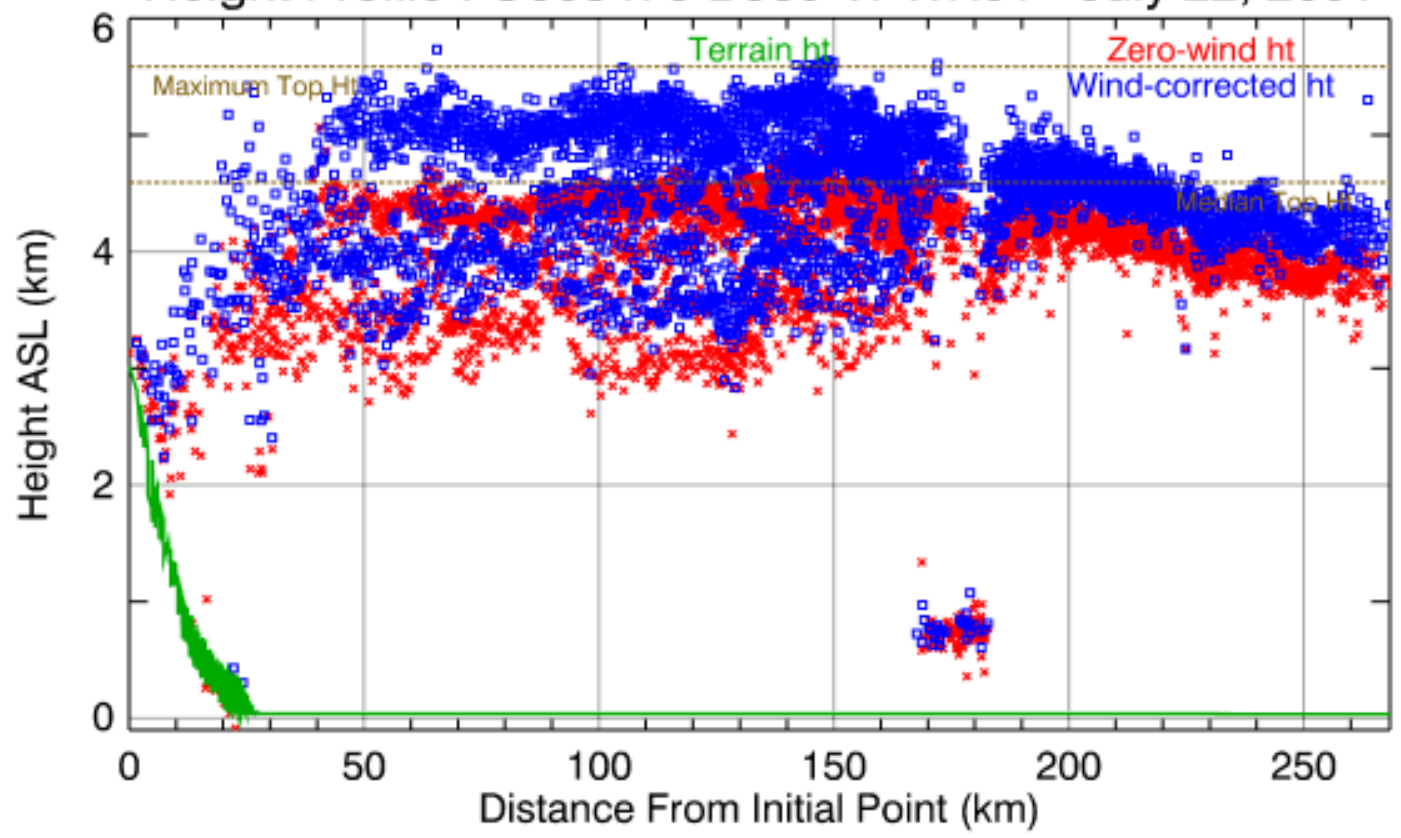

Maximum / Median height estimates: 5.586 / 4.593 km; Retrieval Quality: Good Distance axis origin: lat 37.752 / Ion 14.994

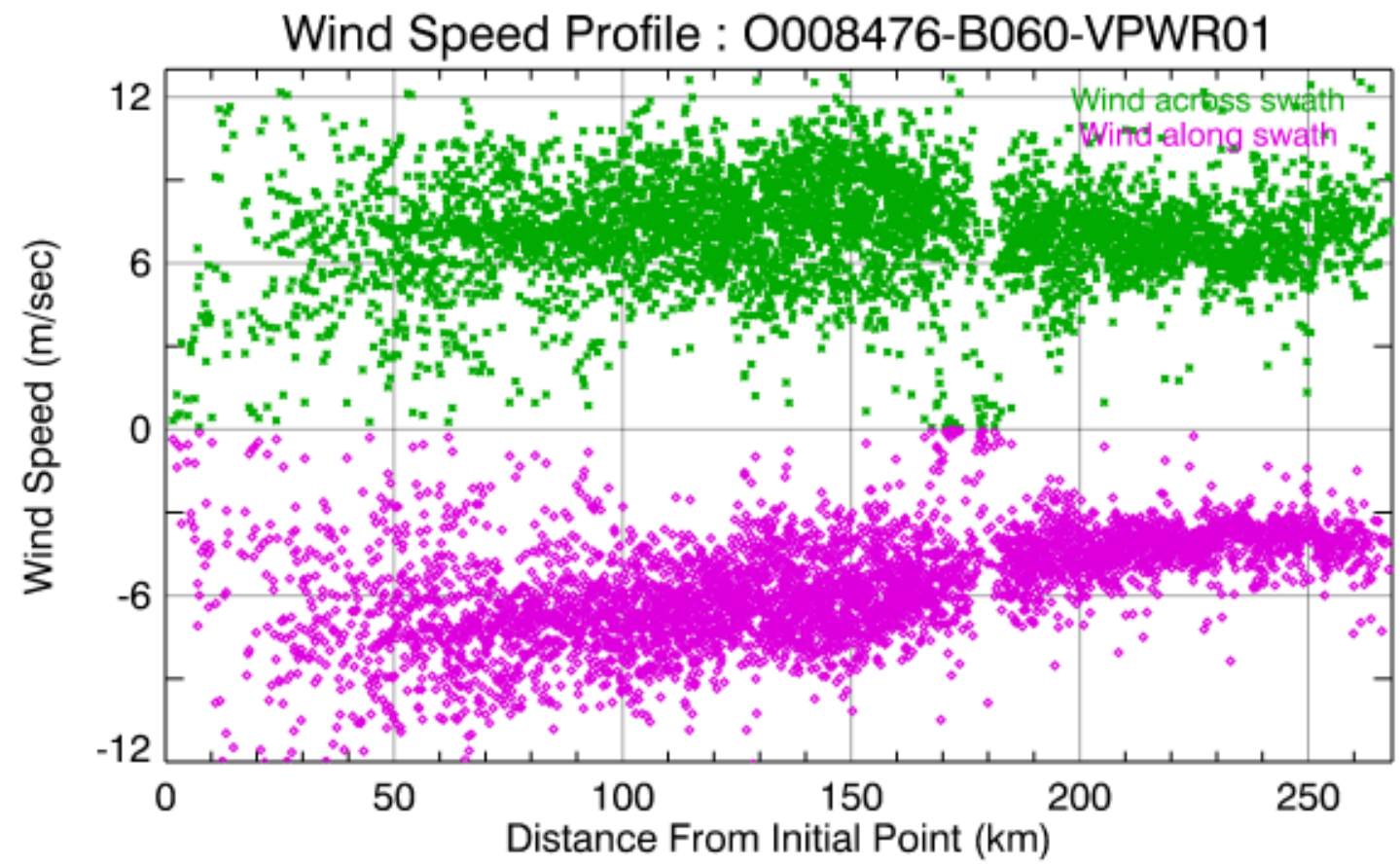



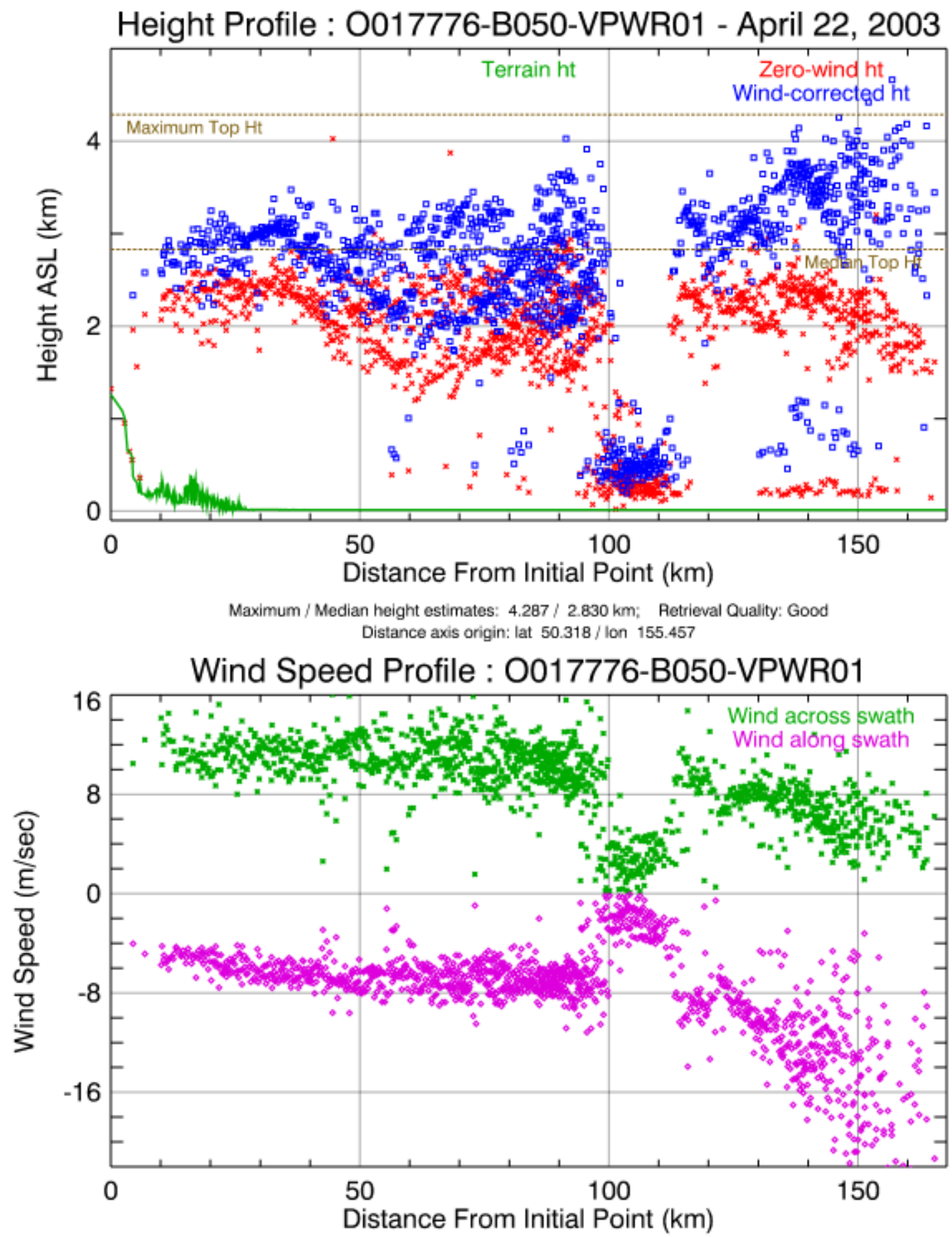